\title{
Cancers, environnements et modes de vie
}

Oléagineux, Corps Gras, Lipides. Volume 11, Numéro 3, 157-66, MAI-JUIN 2004, Nutrition

\author{
Auteur(s) : Elio RIBOLI
}

Chef du Groupe Nutrition et Hormones, Centre International de Recherche sur le Cancer (CIRC), 150 cours Albert Thomas, 69008 Lyon, France

\section{ARTICLE}

${ }^{1} \mathrm{Ma}$ carrière en épidémiologie a commencé un soir vers onze heures quand une amie m'a téléphoné pour m'annoncer que le docteur Franco Berrino, chef du département d'épidémiologie de l'Institut national du cancer de Milan, cherchait un épidémiologiste. J'avais terminé ma médecine et j'étais inscrit en santé publique. Quoique me sentant un peu trop jeune pour ce poste, je me présentai et sollicitai un entretien avec le directeur de I'Institut, le Pr Umberto Véronèse, un spécialiste de réputation internationale (qui allait devenir ministre de la Santé dans les années 2000). II examina mon dossier et, levant les yeux sur moi, il me demanda, étonné : « Mais pourquoi voulez-vous faire de l'épidémiologie ? Votre dossier d'étudiant est excellent ? ». Ainsi en allait-il de notre discipline en 1977, à cette époque considérée comme une voie que l'on empruntait faute de mieux ! C'est dans cet environnement scientifique d'excellence que fut pour moi l'Institut national du cancer de Milan, que j'ai acquis la conviction que la recherche scientifique devait se concevoir de façon multidisciplinaire. C'est ce que j'ai essayé de développer au cours de ma carrière avec plus ou moins de succès. Après une année à l'université Harvard à Boston, où j'ai complété ma formation, j'ai rejoint le CIRC (il y a exactement vingt ans) à l'invitation du Professeur Rodolfo Saracci, un chercheur passionné et rigoureux qui a tant contribué intellectuellement au développement de l'épidémiologie et de la santé publique.Pour en finir avec ce volet biographique de mon exposé, je dois ajouter que le Centre international de recherche sur le cancer de Lyon, qui abrite nos travaux, est un centre des Nations Unies. Ainsi depuis vingt ans, j'ai l'honneur d'être un fonctionnaire des Nations Unies. Aussi, conformément à la règle en vigueur concernant les récompenses personnelles en nature ou en argent, je remettrais le montant de ce prix à l'administration du Centre... laquelle aimablement ouvrira un compte à l'usage de notre laboratoire pour accueillir un jeune boursier.

\section{Les cancers}

Le cancer ne devrait plus s'appeler le cancer. Comme les maladies virales ne s'appellent plus les maladies virales. Personne n'associe le sida au rhume. II y a dans le cancer des pathologies très différentes avec des causes totalement différentes et ce serait l'intérêt de la recherche et de la santé publique, de parler du cancer du poumon, du cancer de l'estomac, du cancer de la peau, etc.

Les figures 1 et 2 présentent les données les plus récentes collectées par notre Centre et donnent une idée de l'impact en terme numérique, en terme de nouveaux cas, diagnostiqués chaque année dans le 
monde : ici le monde a été, de façon manichéenne, grossièrement divisé entre pays économiquement développés et pays économiquement moins développés (une distinction utile sous bien des aspects) et vous voyez comment le cancer du poumon - essentiellement lié au tabac - est aujourd'hui malheureusement très fréquent dans les pays économiquement moins développés ou en voie de développement. Le cancer de l'estomac est également plus fréquent dans ces pays. A l'opposé, le cancer de la protaste est plus fréquent dans les pays économiquement développés, ainsi que les cancers du côlon et du rectum alors que le cancer du foie est plus fréquent dans les pays en voie de développement (dû à l'hépatite et aux aflatoxines...). Le cancer de l'œsophage aussi, et ce n'est pas seulement l'alcool qui en est la cause mais certaines malnutritions que l'on ne connaît pas assez bien. Le cancer de la vessie est plus fréquent dans nos pays, sous l'effet du tabac, certainement, et de certaines expositions professionnelles.

D'autres cancers sont moins fréquents, moins bien connus, peut être moins bien étudiés. Je dois vous signaler ainsi que le lymphome (autre que le lymphome de Hodgkin) augmente depuis 20-25 ans dans les pays développés de façon linéaire... on ne sait pas pourquoi, mais c'est une tendance extrêmement inquiétante. A l'opposé, le cancer de l'estomac est en nette diminution et l'incidence ne représente plus que $10 \%$ de ce qu'il était au début du siècle. II y a donc des changements et chaque cancer évolue selon les modifications de l'environnement et des modes de vie.

Chez la femme, on s'aperçoit combien le cancer du sein, qui était une "prérogative " des pays développés, devient de plus en plus fréquent dans les pays en voie de développement. On constate aussi la grande inégalité face au cancer du col de l'utérus, essentiellement dû au virus du papillome, et donc plus fréquent dans les pays plus pauvres là où les conditions d'hygiène sont les moins favorables.

On voit aussi que le cancer du poumon est maintenant le quatrième cancer chez la femme, "devant " le cancer de l'estomac. Et si l'on s'inquiète parfois beaucoup de la présence dans l'environnement de quelques parties par trillion de certaines substances chimiques, on devrait surtout se préoccuper des milliers de tonnes de goudron que l'humanité inhale chaque jour avec la fumée du tabac.

Pour ce qui relève de la nutrition, deux cancers, le cancer du côlon et le cancer du rectum (je ne vous apprends rien) ont une fréquence beaucoup plus élevée dans les pays développés que dans les pays en voie de développement. Mais du point de vue de la recherche, il est important de noter que ces cancers colorectaux et les cancers du sein sont presque superposables en termes de répartition géographique. Dans tous les pays où l'un de ces cancers est fréquent, les autres le sont aussi. Et c'est pour cela que depuis un certain nombres d'années la recherche se tourne vers l'identification de certains facteurs métaboliques.

L'autre cancer, fortement associé à celui du côlon et du rectum et au cancer du sein, est celui de la prostate, avec une petite exception pour l'Afrique centrale (zone de haute incidence) : c'est peut-être le seul cancer que l'on connaît aujourd'hui pour lequel la prédisposition génétique liée au groupe ethnique est très forte. Ce sont des découvertes récentes : pour le cancer de la prostate, la population noire d'origine africaine présente un risque plus élevé que les populations caucasiennes, elles-mêmes plus exposées au risque de voir se développer ces cancers que les populations du sud-est asiatique. Mis à part cet exemple on pense que globalement c'est plutôt la différence de prédispositions génétiques entre individus, à l'intérieur d'une même population, plutôt qu'entre populations, qui est associée avec le risque de cancer. 
La ( figure 3 ) nous montre combien la fréquence des cancers peut être différente d'une population à l'autre. Ainsi dans un registre du sud de l'Inde (Ambillikai), on voit que le cancer du côlon et le cancer de la prostate sont pratiquement inexistants, ce qui est rarissime. Le cancer du sein commence à augmenter, mais le cancer du col de l'utérus lié à l'infection du virus du papillome y est très fréquent.

Mais tout change, et en l'an 2000, pour la première fois dans l'histoire de l'Inde, on a noté l'apparition d'un plus grand nombre de cas de cancers du sein que de cancers du col de l'utérus à Bombay. Quand la vie économique modifie la vie quotidienne des individus, le risque de cancer se déplace. Parfois dans un sens positif : l'incidence du cancer du col diminue, parfois aussi dans un sens négatif parce que les facteurs de risque ne sont plus les mêmes.

\section{Cancers et mode de vie occidental}

Quand on essaie d'étudier la relation entre mode de vie et cancer, il est inévitable d'associer les cancers les plus fréquents dans nos pays, avec ce que, d'une façon assez vague, on appelle le mode de vie occidental - expression peu précise si on considère que l'on désigne aussi par là le mode de vie des Australiens, qui ne sont pas en Occident. Sommairement on entend sous ce vocable, le mode de vie courant aujourd'hui dans un environnement urbain des pays économiquement développés où existent une large disponibilité de nourriture, et des aliments hautement caloriques, c'est-à-dire riches en calories par rapport au poids qu'ils représentent, riches en graisses et sucres raffinés, en protéines animales (je n'ai rien contre les protéines animales, mais elles caractérisent aussi notre alimentation) à quoi il faut ajouter un mode de vie plus sédentaire, une consommation de tabac, d'alcool, etc. Paramètres dont les conséquences directes sont également l'augmentation de la taille à l'âge adulte, un âge plus précoce des premières règles, une présence croissante de l'obésité... Ce mode de vie a-t-il un impact sur le diabète, les maladies cardio-vasculaires, l'hypertension, les cancers ? Ce sont évidemment les questions que l'on ne peut pas ne pas se poser.

La taille: je ne vous apprends rien, à vous nutritionnistes, en vous rappelant que les Hollandais constituent aujourd'hui la population dans le monde dont la taille moyenne est la plus élevée. Selon les données relevées à partir de 30000 sujets, aux Pays-Bas la taille moyenne des hommes de moins de 40 ans est de 1,86 m, alors qu'elle était de 1,65 m pour les conscrits il y a 150 ans ( (figure 4) ). Sans changement génétique connu, la taille a ainsi augmenté de $21 \mathrm{~cm}$. Et il en va de même pour les Danois, les Suédois et les Norvégiens. C'est ce que certains collègues ont appelé un apport énergétique et protéique au maximum de la taille " génétiquement prévu ", l'idée étant que chacun $d^{\prime}$ 'entre nous selon ses gènes et son équilibre métabolique, dispose d'une taille maximale potentielle qu'il atteindra s'il mange suffisamment entre la naissance et ses 19-20 ans. C'est un changement considérable si vous pensez qu'il a fallu un demi-million d'années à l'espèce humaine pour devenir ce qu'elle est génétiquement, et seulement 150 ans pour voir sa taille augmenter de $21 \mathrm{~cm}$.

L'âge des premières règles ( (figure 5) ) : alors qu'il était tout à fait normal, il y a 150 ans, en Norvège, en Finlande, en Allemagne, en Suède que les premières règles en moyenne surviennent autour de dixsept ans, aujourd'hui on demande aussitôt une consultation chez l'endocrinologue si les jeunes filles ne sont toujours pas réglées à cet âge... alors que bien sûr, les gènes n'ont pas changé. Or on constate que l'arrivée des règles après dix-sept ans est associée à une diminution de $40 \%$ du risque de développer un cancer du sein ( (figure 6) ). Autrement dit, dans un pourcentage élevé des cas, le cancer du sein peut être expliqué exclusivement par les changements d'alimentation, des conditions d'hygiène et des modes de vie, survenus avant douze ans ( (figure 7) ). Ce qui évidemment limite 
l'effet des politiques de prévention au-delà de 40 ans. Ces considérations sont dérangeantes mais vraies et nous obligent à remettre en cause les affirmations que l'on entend toutes les cinq minutes dans les médias.

Prévalence de l'obésité : on est plus grand, les jeunes filles sont réglées plus tôt, le jeune homme, pour ce que l'on sait, présente une maturation sexuelle un peu plus précoce, mais pas tellement plus précoce que les jeunes femmes (on ne sait pas pourquoi)... alors que l'augmentation de l'obésité est absolument épidémique. En France, cela ne se remarque pas beaucoup, mais c'est l'expérience de tous ceux qui ont voyagé aux Etats-Unis : l'augmentation de l'obésité y est au-delà de l'imaginable. Quand je faisais médecine on parlait pour certains cas d'une obésité "monstrueuse " nécessitant I'hospitalisation du sujet en vue d'un traitement hormonal, métabolique... aujourd'hui un quart de la population américaine a des difficultés pour s'asseoir dans un siège de classe économique d'un avion... c'est aussi dramatique que cela.

Où va-t-on ? Voici les projections de la prévalence de l'obésité entre 2000 et 2030 en Angleterre, aux Etats-Unis, à l'lle Maurice, en Australie, et au Brésil ( (figure 8) ). L'épidémie d'obésité dans les pays en voie de développement, au sein de populations qui n'ont pas eu le temps de s'adapter à une richesse des aliments provenant de l'agriculture, est absolument impressionnante.

Ce diagnostic était déjà posé il y a une quinzaine d'années, et il fut au point de départ de notre décision, au Centre international de recherches sur le cancer (CIRC), d'étudier et de mieux connaître les relations existant entre les différents cancers et les modes de vie. Car s'il est facile de constater empiriquement cette relation, il est beaucoup plus difficile de l'étudier, chaque paramètre influençant certainement les autres.

C'est ici qu'est intervenue la dimension européenne de nos travaux. Avec Rodolfo Saracci - alors directeur du service d'Epidemiologie au CIRC - nous nous sommes dits qu'il nous fallait " profiter » de l'Europe, profiter du fait qu'il existait en Europe une grande variabilité de consommation alimentaire et de modes de vie associée à une grande variabilité de l'incidence du cancer - je vous rappelle qu'en Murcia (sud de l'Espagne), l'incidence totale du cancer est le tiers de celle de Glasgow, ville située à une heure trente minutes de vol : le tiers cela signifie que si les Écossais vivaient comme les Murciens, il y aurait parmi eux chaque année $66 \%$ de cas de cancer de moins, soit pour des dizaines de milliers de personnes beaucoup de souffrances épargnées.

Nous pouvions aussi mettre à contribution le tissu européen de la recherche, en médecine, biologie, nutrition, de toute première qualité - nous ne devons pas, et nous n'avons aucune raison de nourrir un complexe d'infériorité à l'égard de nos amis d'Amérique du Nord, nous avons en Europe les connaissances, les hommes, la technologie... et si parfois manque la volonté politique nous avons tout le reste pour faire de la bonne recherche médicale.

Ainsi nous avons monté l'étude EPIC (European prospective investigation into cancer and nutrition) qui a consisté à réunir des équipes de chercheurs de dix pays, pour collecter les données sur 521468 sujets (chaque sujet nous ayant coûté une petite goutte de sueur), et prélever environ 400000 échantillons sanguins. Collecter du sang sur cette échelle n'eut rien d'évident (figures 9-11). Quand en 1990, je proposai devant le conseil scientifique du CIRC l'idée de créer une banque biologique, une partie des membres du conseil, des éminents scientifiques, des collègues que je respecte et avec lesquels j'ai toujours gardé d'excellents rapports - s'y étaient opposés. Nous n'avions 
pas d'hypothèse! Nous demandions des budgets alors que nous ne savions pas quelles hormones, quels bio-marqueurs nous souhaitions mesurer ! J'ai fait valoir, évidemment, que c'était en raison même de cette ignorance, de ce qui serait utile en 2010, compte tenu des progrès d'ici là de la recherche fondamentale en biologie, en biochimie, en génétique, qu'il nous fallait constituer cette banque biologique.

Finalement, ce sont huit millions d'échantillons stockés dans l'azote liquide qui sont aujourd'hui à notre disposition, chaque cuve contenant 135000 échantillons. Soit une utilisation du volume excellente. Pour stocker ces cuves, il a fallu construire un bâtiment ( (figure 11) ), ce qui a été fait grâce à des legs reçus par le CIRC, en particulier des familles lyonnaises.

\section{L'étude prospective}

Qu'est-ce qu'une étude prospective? Une étude prospective se base sur une idée simple: son principe consiste à observer ce qui se passe dans l'axe du temps, après avoir collecté au départ toutes les informations possibles, sur les habitudes alimentaires, les données anthropométriques (taille, poids, tour de hanche) de tous les sujets, sur lesquels ont été effectués également un prélèvement sanguin. Le sang est fondamental car l'utilisation des bio-marqueurs est beaucoup plus claire du point de vue de la logique biologique si les bio-marqueurs sont identifiés avant l'apparition du cancer, plutôt que mesurés après son apparition ( (figure 12) ).

II nous a fallu ainsi environ dix ans pour créer cette base de données, cette banque biologique. Le réseau européen de registres du cancer nous ayant permis d'effectuer un suivi très efficace, croisements des fichiers informatiques, presque partout sauf en Grèce, en France et en Allemagne où la distribution géographique de la cohorte ne le permet pas.

L'objectif final était de mettre en évidence une relation entre l'apparition de la maladie et ce que l'on a collecté et mesuré au départ, afin de déterminer dans quelle mesure ceux qui développent un cancer particulier sont différents ou ne sont pas différents des sujets du même sexe, de même âge, vivant au même endroit, dans la même ville, mais restés indemnes de cancer. C'est ce qu'on appelle "l'étiologie prospective".

Pourquoi était-il nécessaire de réunir les données sur un demi-million de sujets ? Parce que chaque cancer est une maladie rare. II fallait un demi-million de sujets dont 300000 femmes pour observer environ 6000 cas de cancer du sein, 2000 cas de cancer du côlon, 1500 cas de cancer de la prostate, etc. Pour étudier prospectivement une pathologie comme le cancer, il faut aller au-delà du nombre total de cancers, chacun ayant une logique spécifique il faut donc investir...

\section{Une étude multicentrique}

Petite diversion pour préciser pourquoi à la fin des années 80 nous avons souhaité faire une étude multicentrique .

En épidémiologie on procède un peu comme au sein des laboratoires de la recherche fondamentale avec les souris... seulement il y a une petite différence. En laboratoire, les souris issues d'une même 
lignée sont identiques et seules les doses varient, le travail consistant à mesurer leurs effets respectifs. En épidémiologie, l'étude doit porter sur des individus différents eux-mêmes soumis à des expositions différentes. Le problème étant qu'à l'intérieur d'une population il est plutôt improbable de rencontrer de nombreux sujets soumis à des expositions très différentes.

C'est ce que Geoffrey Rose, un des pères britanniques de l'épidémiologie moderne dans les années 1970, a appelé les déterminants de l'incidence d'une maladie. Soit l'exemple de l'hypertension. Une étude très importante a été conduite sur ce sujet à Londres dans les années 60-70, la London Civil Servants Study. Parallèlement, les nutritionnistes disposaient d'une étude sur la tension artérielle au sein d'une population de nomades, du Kenya. Chez les Londoniens les résultats montraient une prévalence très élevée de sujets présentant une hypertension systolique (au-delà de 14), soit un phénomène rarissime au Kenya. L'étude Kenya seule n'aurait jamais permis d'identifier la cause de I'hypertension à 18. Inversement si on s'était limité aux seuls Londoniens on n'aurait jamais pu comprendre qu'en réalité une tension normale est autour de 11 et non pas 13-14 ( (figure 13) ).

Ainsi si l'on veut étudier l'impact des facteurs liés aux populations, il faut pouvoir étudier en même temps les populations et les individus. Ce n'est que lorsque l'on peut faire ces deux choses qu'on a de bonnes chances d'identifier les facteurs véritablement causaux.

Je voudrais dire en passant que cette façon d'étudier les problèmes, uniquement à l'intérieur d'une population (en prenant juste les Londoniens, les Parisiens, les Marseillais, les Milanais), a eu un impact délétère sur le concept de normalité en médecine. Pour le dire avec brutalité, on peut affirmer que de nombreux concepts de normalité sont tout simplement faux : on confond ce qui est fréquent avec ce qui est physiologiquement normal : ce qui n'est pas la même chose. D'où ce paradoxe, formulé par Geoffrey Rose en 1985 : si on avait conduit les études sur le cancer du poumon uniquement au sein des populations de fumeurs d'un paquet de cigarettes par jour, on serait arrivé à la conclusion inévitable que le cancer du poumon est une maladie génétique! Ce qui dans ces conditions aurait été tout à fait exact parce que la génétique aurait été alors la seule variante entre les individus développant ce type de cancer et les autres ! Ce qui peut prêter à sourire mais n'est pas banal du tout!

La variabilité de l'exposition est fondamentale si on veut mettre en lumière une relation entre exposition et risque de maladie. Donc l'idée développée avec l'étude EPIC était que, plutôt que de continuer à opposer étude à niveau individuel et étude au niveau écologique, il convenait de combiner les deux et d'essayer de prendre avantage du fait qu'il y a une composante de la relation entre exposition et maladie qui peut être vue seulement au niveau géographique et une composante qui peut seulement être vue à l'intérieur de chaque population. L'hétérogénéité est donc fondamentale. Ce que nous appelons en termes techniques l'hétérogénéité de l'exposition.

C'est ce que nous avons essayé de mettre en lumière dans un numéro spécial du journal Public Health Nutrition (2002; $5: 1111-1345 S)$ récemment dédié à EPIC. Nous avons mesuré l'alimentation à l'aide d'un questionnaire spécifique à chaque pays pour l'ensemble de la cohorte (521 000 individus) et pour 39000 d'entre eux on a remesuré leur alimentation avec un rappel de 24 heures, standardisé... un travail énorme! Un jour nous publierons les recettes (nous en avons collectées plusieurs milliers dans différentes langues). 
Ces deux méthodes nous ont permis d'étudier et de caractériser assez bien les consommations alimentaires au niveau des populations. Les résultats ont été plutôt rassurants quant aux variations du niveau de consommation. On a constaté par exemple que la consommation de fruits est très élevée dans le sud de l'Europe (Ragusa en Sicile, Murcia, San Sebastien, Turin, Granada, Oviedo, Varèse) et moitié moins élevée dans le nord (Suède, Norvège, Pays-Bas) ( (figure 14) ). En Angleterre, on constate la différence entre les sujets représentant la population générale et les végétariens. On a donc un gradient très impressionnant de populations : c'est ce que l'on espérait trouver.

On constate également un gradient très impressionnant pour la consommation de légumes ( (figure 15) ). Mais l'observation d'un aliment à la fois ne nous informe pas sur le cadre global, ce que nous avons appelé le modèle alimentaire. Nous avons publié cette série de représentations graphiques où le cercle est la moyenne européenne et la ligne jaune verte - jaune pour les femmes et verte pour les hommes - indique de combien diverge un pays par rapport à notre étude d'un demi-million de sujets.

Vous voyez que la population générale en Angleterre consomme beaucoup de boissons gazeuses, de soft drink, nous avons fait la découverte bouleversante que les Anglais boivent du thé, mais aussi qu'ils consomment beaucoup de sucre et de sucreries, peu d'huiles végétales, beaucoup de lait : soit un modèle alimentaire très caractérisé ( (figure 16) ). Si on prend les végétariens anglais on voit que, tout en se réclamant d'une autre approche de la nourriture, ils continuent à consommer beaucoup de sucre et de sucreries, beaucoup de margarine, pas du tout d'huile d'olive, plus de beurre que la moyenne, beaucoup de thé et effectivement pas de charcuterie ni de viande ( (figure 17 )).

On s'aperçoit que les Allemands consomment énormément de beurre, de saucisses (autre grande découverte), peu d'huile d'olive, moyennement de sucre et de soft drink et beaucoup de jus de fruit ( (figure 18) ). Les Italiens consomment des pâtes et du pain, des fruits, très peu de beurre, modérément du lait et très peu de café : la consommation y est le cinquième de ce qu'elle est au Danemark par habitant (car les cafés y sont servis très courts) ( (figure 19) ). En Espagne, on constate une grande consommation de légumineuses, de haricots (une caractéristique qui a été exportée en Amérique latine), de poisson, d'huile d'olive ( (figure 20) ). Chaque pays a vraiment une image propre. La France a son image et à l'intérieur de la France on retrouve les différences des pays qui l'entourent ( (figure 21) ). Dans le Sud, peu de beurre, beaucoup plus dans le Nord, plus de poisson au bord de I'Atlantique, apparemment au Nord (effet de la proximité de l'Angleterre) on y boit plus de thé.

En plus des données provenant des questionnaires, EPIC compte sur l'information additionnelle et indépendante fournie par les marqueurs biologiques. Le lycopène, par exemple, est un excellent marqueur de la consommation de tomates ( (figure 22) ). Le graphique montre le niveau sanguin très élevé de lycopène mesuré en Sicile et dans le reste de l'Italie par rapport au niveau très bas en Suède et au Danemark. Pour l'eicosapentanoïque (EHA), excellent marqueur de la consommation de poisson, le Danemark, la Suède, l'Angleterre et l'Espagne du Nord ont des niveaux très élevés, et les végétariens, Florence, Milan et Ragusa des niveaux bas ( (figure 23) ). Donc c'est l'image à l'envers : les pays qui étaient en haut pour les tomates, sont en bas pour le poisson. Cela signifie que si les deux sont bons dans les deux cas, il faudrait faire un effort pour améliorer l'alimentation. 


\section{Les fibres}

C'est un sujet très complexe. Comme vous le savez, l'hypothèse biologique était très forte, les mécanismes de protection des fibres avaient été beaucoup étudiés. De nombreuses équipes avaient travaillé sur cette hypothèse et beaucoup de données avaient été réunies appuyant cette idée qu'une alimentation riche en fibres dans le long terme protège contre le cancer du côlon et du rectum. Puis, une série d'études conduites essentiellement en Amérique du Nord, est venue invalider ou tout au moins n'a pas confirmé cette relation entre consommation de fibres et protection contre le risque de développement de cancer du côlon, ce qui a conduit il y a deux ou trois ans à presque retirer I'hypothèse de la scène scientifique.

Il y a quelques mois nous avons publié les premiers résultats de l'étude EPIC sur la consommation de fibres ( (figure 24) ). En profitant de cette grande variabilité, on a pu comparer des individus consommant en moyenne $13 \mathrm{~g}$ par jour, et les derniers $38 \mathrm{~g}$ par jour. Ce qui est intéressant c'est que sur la base d'un demi-million de sujets, nous trouvons bien une relation de diminution du risque du cancer du côlon et du rectum avec une augmentation de la consommation de fibres. Ce qui est encore plus intéressant, c'est qu'en réalité en prenant ce point sur la médiane, on peut en déduire qu'environ la moitié de la population européenne a déjà une protection de l'ordre de 20-30 \% mesurée sur cette échelle du questionnaire d'origine. Si on introduit une correction pour l'imprécision, on obtient ainsi cette ligne verte, qui nous dit qu'en consommant environ 30-35 g de fibres par jour on devrait normalement, dans le long terme, réduire de 40 \% l'incidence du cancer du côlon et du rectum.

Pourquoi les études américaines présentent-elles des résultats différents ? Si on considère ces études, la Nurses Health Study (90000 infirmières), Health Professionals Study (50000 médecins), ainsi qu'une étude en Suède, on s'aperçoit que pour les fibres céréales qui tiennent une place prépondérante dans notre étude le niveau élevé des infirmières américaines est équivalent au niveau bas de l'étude EPIC : évidemment s'il faut consommer $12 \mathrm{~g}$ de céréales par jour pour avoir une protection on ne risque pas de l'obtenir si la consommation la plus élevée est de $5 \mathrm{~g} / j o u r$. Et on revient au concept déjà évoqué de population : les populations et les individus. On ne peut tout étudier dans une seule population!

Notre étude a été publiée en parallèle dans le Lancet avec une étude de nos collègues de l'Institut national du cancer des Etats-Unis, qui ont étudié les polypes chez 70000 individus et ils ont trouvé une relation semblable, c'est-à-dire une consommation élevée de fibres associée avec $25 \%$ de réduction et qui remet en question les conclusions précédentes.

Dans la même étude, nous avons trouvé une association qui n'est pas inattendue avec viande et charcuterie ( (figure 25) ). Nous avions auparavant avec Teresa Norat travaillé sur une réanalyse des précédentes études épidémiologiques sur les viandes, les poissons et les charcuteries en relation avec le cancer du côlon. Et les résultats montrent une augmentation de 20 à $30 \%$ du risque de développer un cancer du côlon chez les sujet situés au niveau le plus élevé de consommation de viande et de poisson et une augmentation de $30 \%$ du risque du cancer du côlon pour une consommation élevée de charcuterie, tandis que les volailles sont sans effet sur le risque. C'est ce que l'on trouve dans l'étude EPIC, et nous sommes sur le point de publier ces résultats, donnés ici en avant première. On trouve une confirmation d'un effet de protection apparente de la consommation de poisson, une petite augmentation mais à la limite de la significativité, quand on consomme au-delà de $80 \mathrm{~g} / \mathrm{jour}$ (soit une consommation quotidienne de viande ou de charcuterie) du risque de cancer du côlon. Comment expliquer ces résultats? Aucune hypothèse parmi celles aujourd'hui proposées n'est 
démontrée. Le contenu en hème, en fer et en myoglobine, la formation endogène des composés nitrosés dans le côlon, les hypothèses ne manquent pas...II s'agit donc de quelque chose qu'il faut étudier pour voir si c'est vraiment la consommation de viande et de charcuterie qui est en cause ou d'autres facteurs qui se cachent derrière.

Pour l'heure nos résultats nous conduiraient à penser que ce sont effectivement les consommations de viande et de charcuterie qui sont en cause. Ces résultats viennent pratiquement de sortir de l'ordinateur. Si on prend une consommation modeste de viande (moins de $30 \mathrm{~g} / \mathrm{jour}$ ) et élevée de fibres (au-delà de $30 \mathrm{~g}$ par jour) comme référence, on s'aperçoit que ceux qui consomment très peu de fibres et beaucoup de viande ont un risque augmenté de $50 \%$. II y a donc un effet dose, en deçà d'un certain niveau de fibres consommé, la consommation de viande est associée à une augmentation du risque. La seule bonne nouvelle serait que lorsqu'on consomme beaucoup de fibres, il pourrait ne pas y avoir d'effet de la consommation de viande. Tout cela est à étudier.

Même problème avec le poisson associé à une réduction du risque. Si on considère les sujets consommant beaucoup de fibres et de poisson, on observe qu'une consommation faible en fibres et en poisson augmente de $70 \%$ le risque de développer un cancer du côlon avec une tendance à la linéarité dans chacun des groupes dans les deux sens.

En épidémiologie on a tendance à dire que ces deux facteurs sont indépendants.

Au-delà de l'alimentation, de nombreux autres facteurs interviennent dans la physiologie des cancers en général et du cancer du côlon en particulier, par exemple l'obésité et l'indice de masse corporelle. La plupart des études montrent une augmentation du risque de développer un cancer du colorectum pour la plupart des individus présentant un IMC élevé et une réduction du risque de 20 à $40 \%$ pour les sujets ayant une activité physique élevée par rapport à une vie sédentaire.

Qu'il y a-t-il derrière ça ? Et bien nous avons pensé qu'il pouvait y avoir l'insuline et le problème de l'insulino-résistance. Nous avons publié il y a deux ans la première étude mettant en relation un marqueur assez bon de l'insulino-résistance (le peptide C) et l'augmentation du risque de développer un cancer colorectal - étude sur 15000 femmes de l'Etat de New York suivies pendant 10 ans et mesurées à partir d'échantillons collectés au départ. Le cancer du côlon de plus en plus se différencie du cancer du rectum, non seulement parce qu'il est plus fréquent chez les hommes que chez les femmes, mais parce que les facteurs métaboliques sont plus étroitement associés au côlon qu'au rectum.

Pour conclure sur la complexité d'étudier l'environnement, les modes de vie et les cancers : si d'un côté des facteurs sont identifiés qui apparemment augmentent le risque, comme l'obésité, l'insulinorésistance, l'alcool (qui induit une augmentation du risque du cancer du côlon et du rectum de $50 \%$ ), les viandes et les charcuteries, d'un autre côté les aliments riches en fibres, les fruits, les légumes, les poissons et l'activité physique apparaissent comme des aliments et des comportements protecteurs. Est-ce que nous sommes tous égaux devant les cancers ? C'est le programme que nous nous sommes fixés. 
De nombreuses informations sont données sur notre site internet : http://www.iarc.fr/epic/ et sur le site du Consortium de Cohortes du National Cancer Institute (Bethesda, Etats-Unis): http://epi.grants.cancer.gov/Consortia/cohort.html.1 Texte de la conférence prononcée, lors de la remise du prix de l'Institut français de nutrition, le 9 décembre 2003 à Paris.

Illustrations

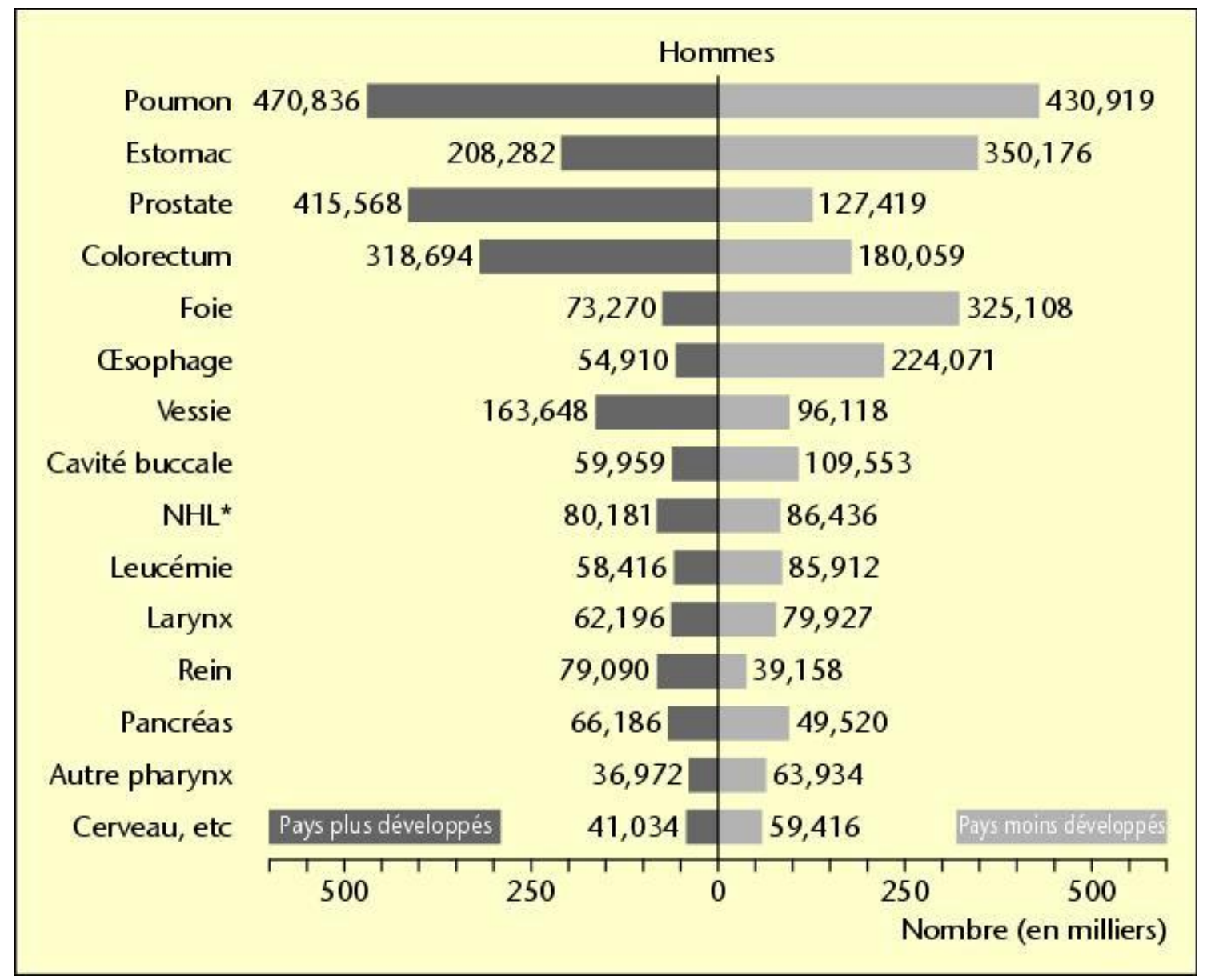

Figure 1 Incidence annuelle des différents cancers chez les sujets masculins. ${ }^{*} \mathrm{NHL}$ : Iymphome non hodgkinien. 


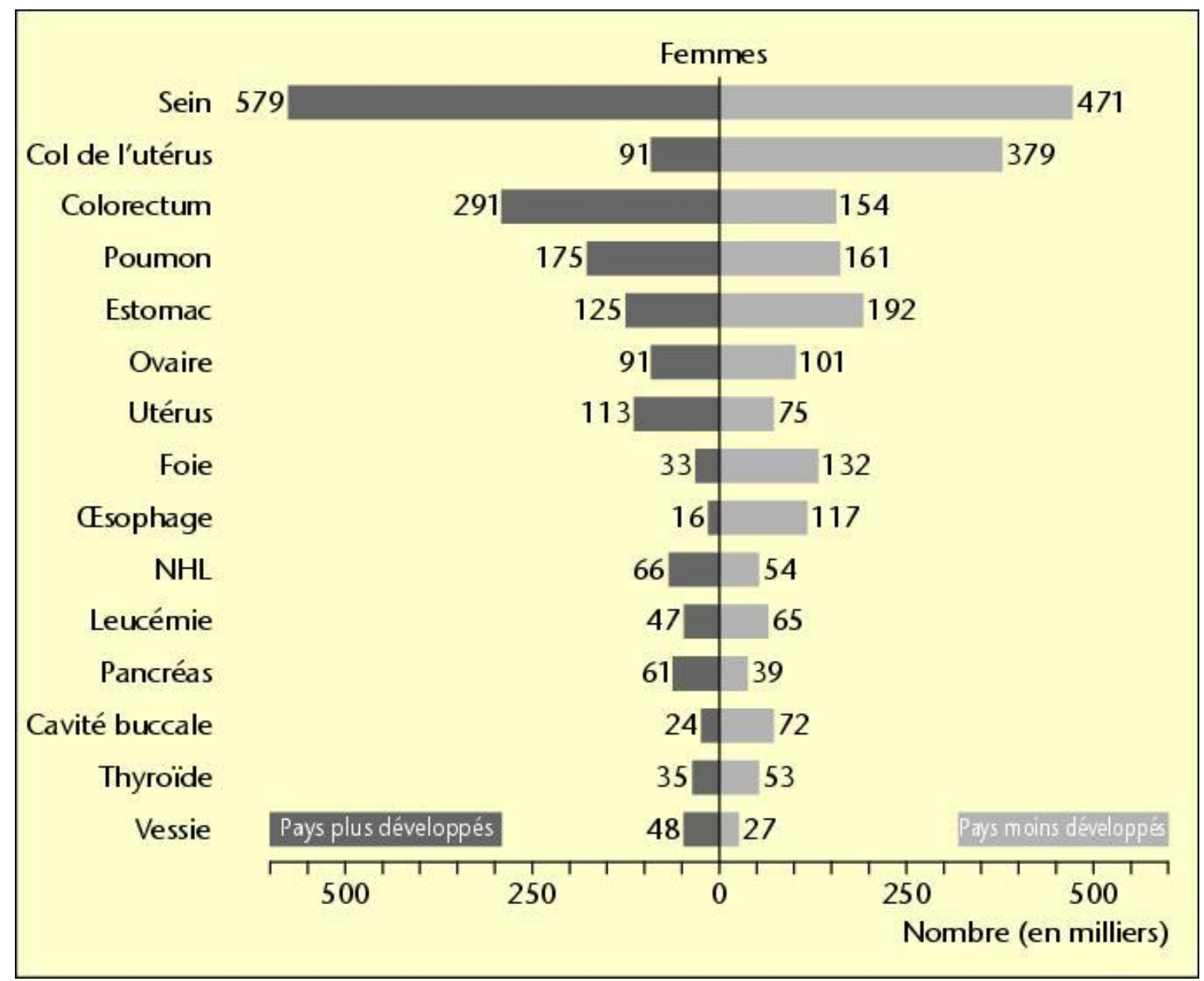

Figure 2 Incidence annuelle des différents cancers chez les sujets féminins.

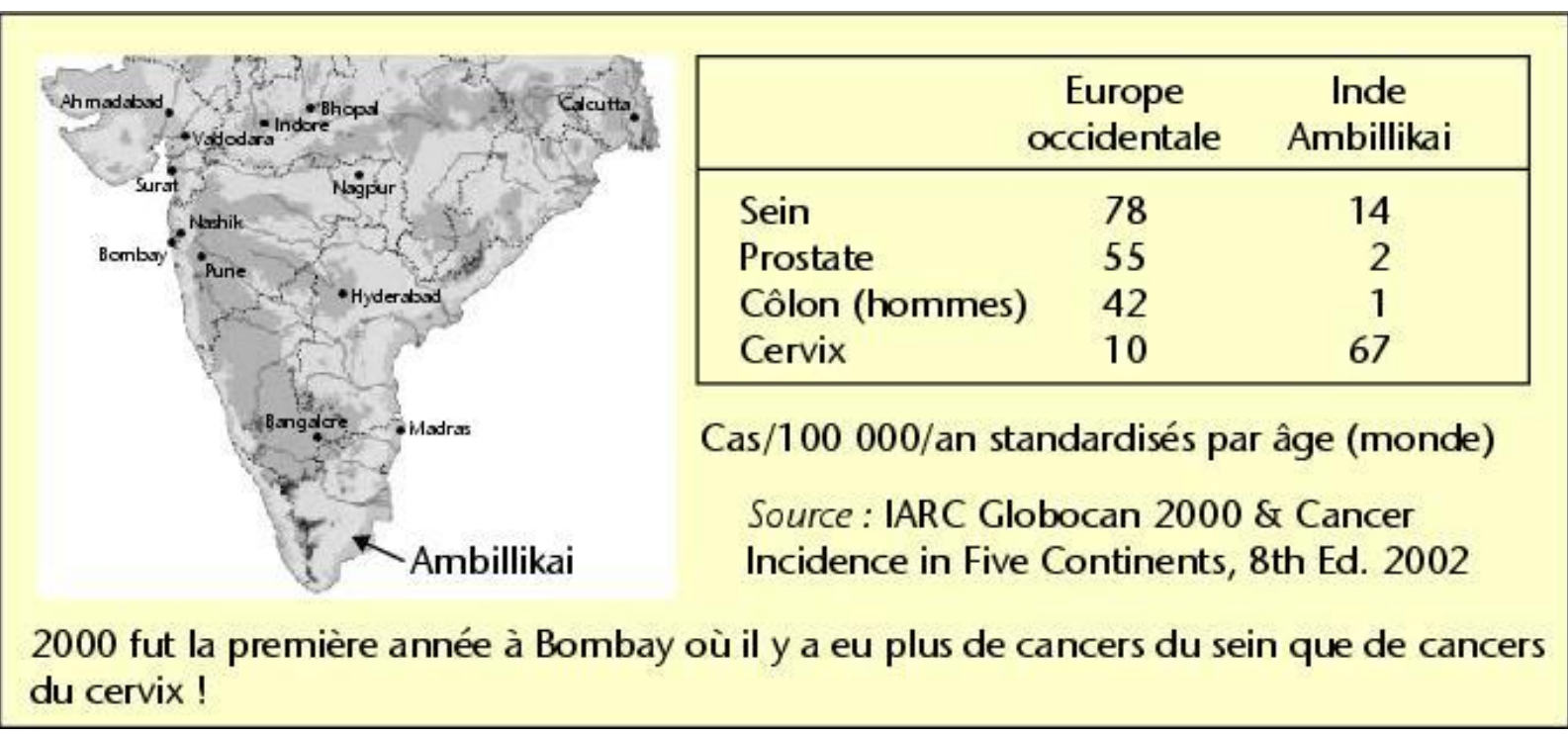

Figure 3 Incidence annuelle des différents cancers en Inde. 


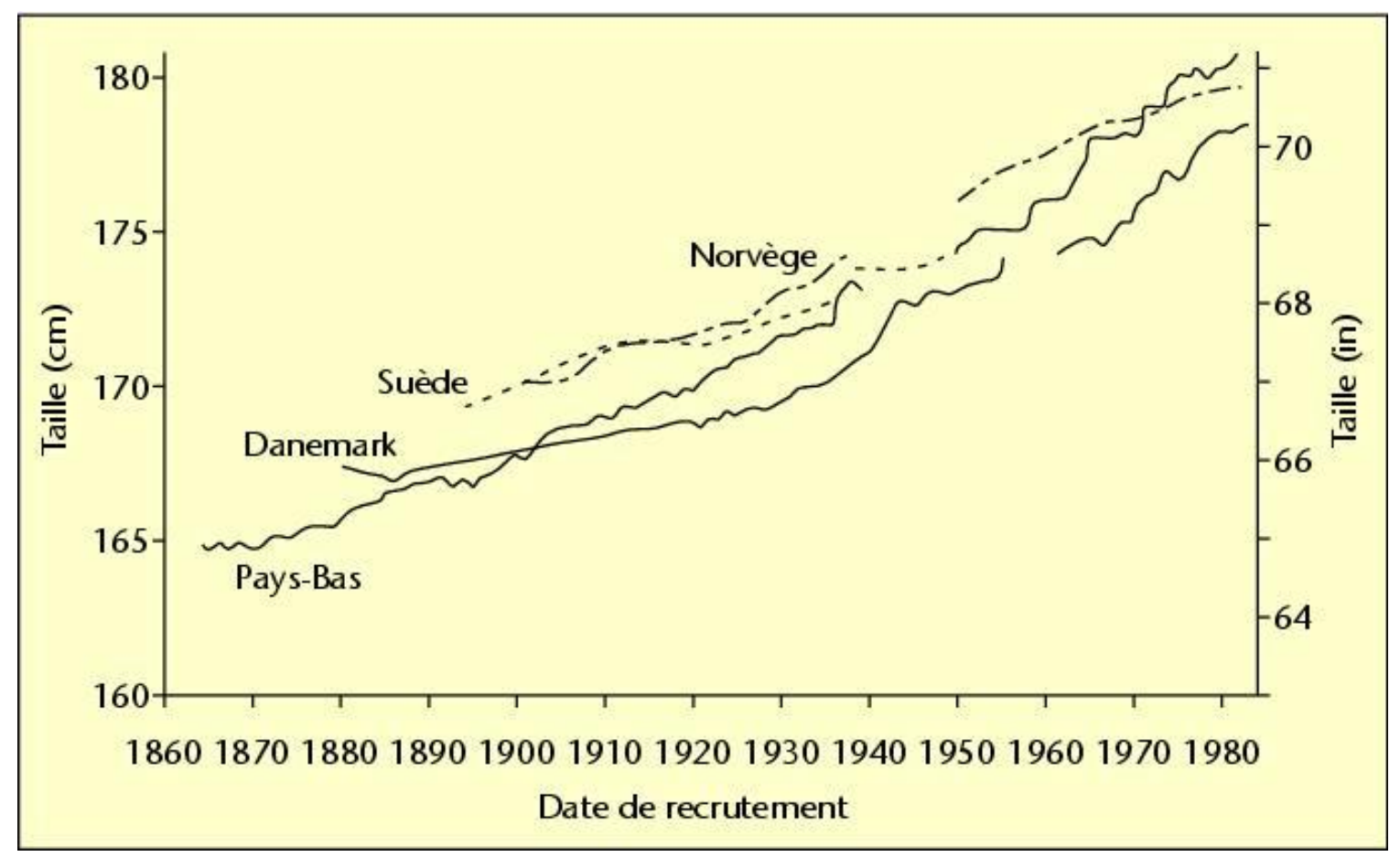

Figure 4 Evolution de la taille des sujets masculins dans plusieurs pays.

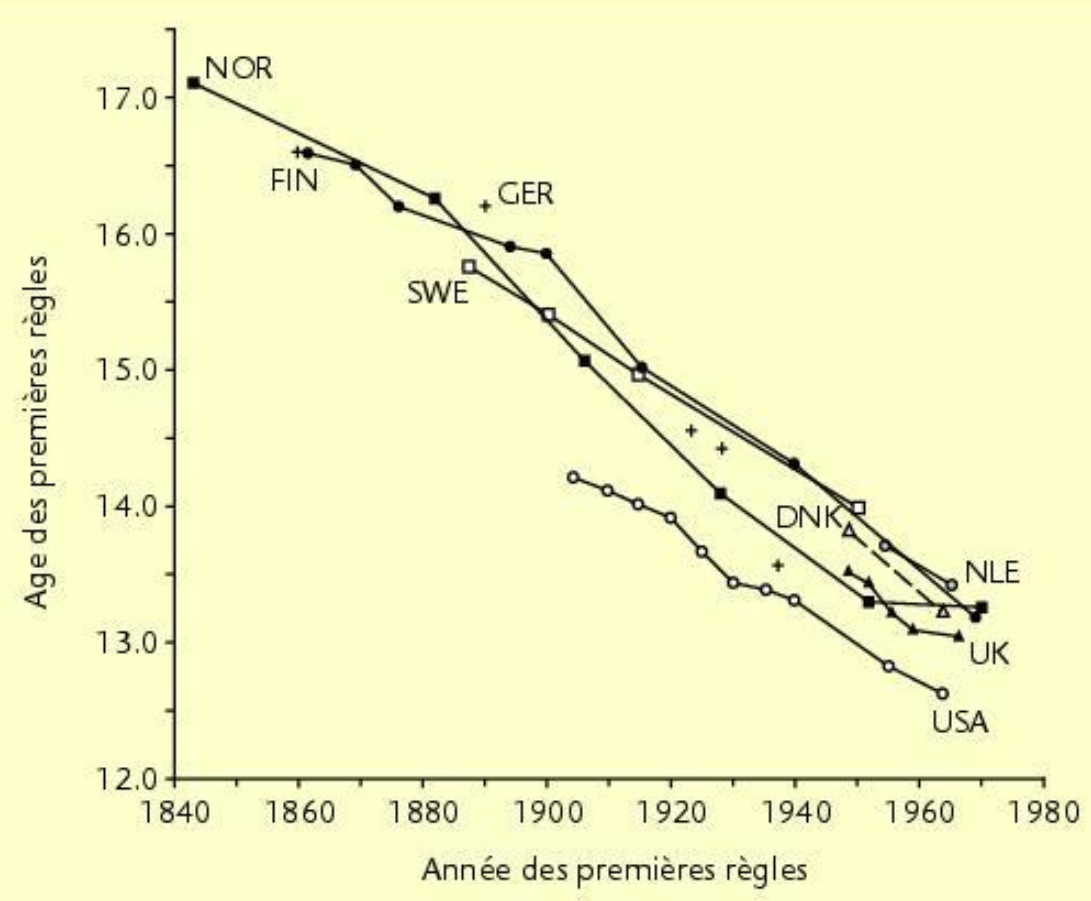

Figure 5 Evolution de l'âge des premières règles. 


\begin{tabular}{|cc|}
\hline Age des premières règles & OR $(95 \% \mathrm{Cl})$ \\
\hline$\leq 12$ ans & 1.0 (référence) \\
13 & $1.1(0.8-1.5)$ \\
14 & $0.9(0.7-1.2)$ \\
15 & $0.9(0.7-1.3)$ \\
16 & $0.8(0.6-1.1)$ \\
$\geq 17$ & $0.6(0.5-0.9)$ \\
\hline
\end{tabular}

Figure 6 Risques d'apparition de cancer du sein et âge des premières règles.

\begin{tabular}{|lcccc|}
\hline Type de cancer & $\begin{array}{c}\text { \% d'augmentation du risque } \\
\text { versus poids normal } \\
\text { Surcharge pondérale } \\
\text { (INC 25-30) }\end{array}$ & $\begin{array}{c}\text { Cbésité } \\
\text { (INC > 30) }\end{array}$ & $\begin{array}{c}\text { Cancers } \\
\text { attribuables }\end{array}$ & $\begin{array}{c}\text { Nouveaux cas/an dus } \\
\text { à un excès de poids }\end{array}$ \\
\hline Endométre & 49 & 152 & $39 \%$ & 14000 \\
Rein & 36 & 84 & $25 \%$ & 10000 \\
Vésicule biliaire & 34 & 78 & $24 \%$ & 6000 \\
Côlon & 15 & 33 & $11 \%$ & 21000 \\
Sein & 12 & 25 & $8 \%$ & 13000 \\
Prostate & 16 & 12 & $4 \%$ & 5000 \\
\hline
\end{tabular}

Figure 7 Excès de poids et cancers dans l'Union européenne. 


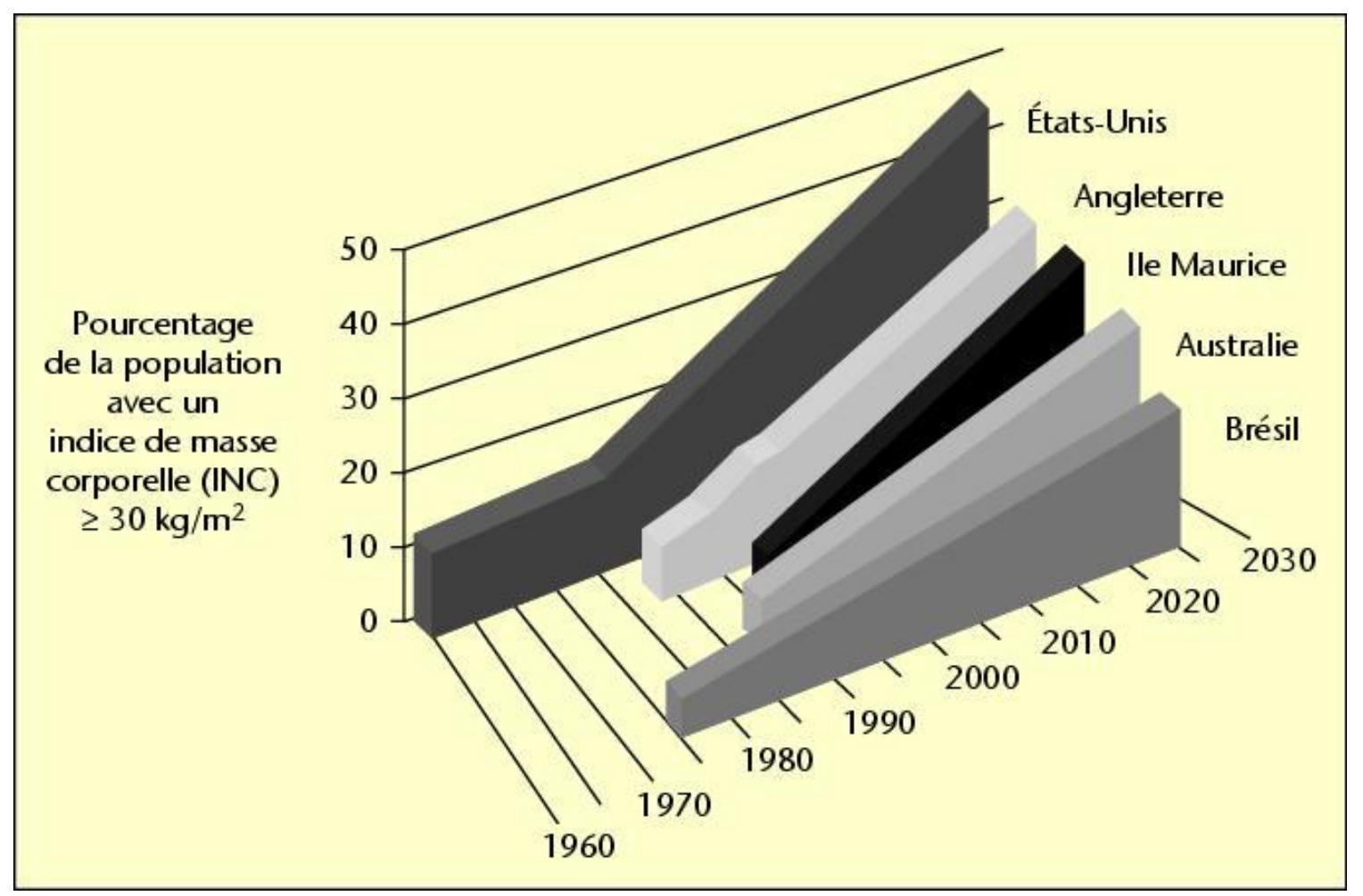

Figure 8 Evolution de l’obésité dans le monde.

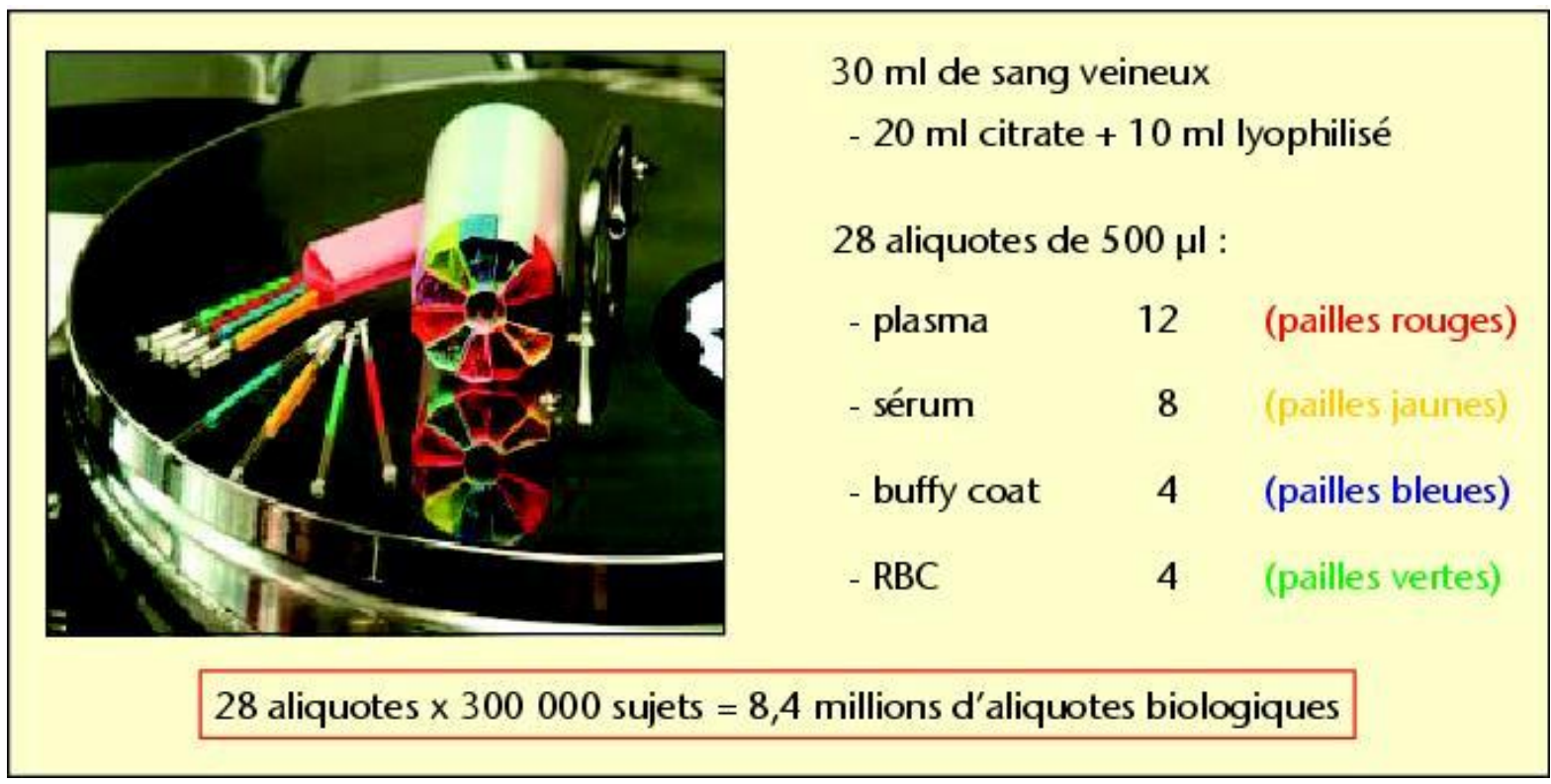

Figure 9 Collecte et stockage des échantillons sanguins. 


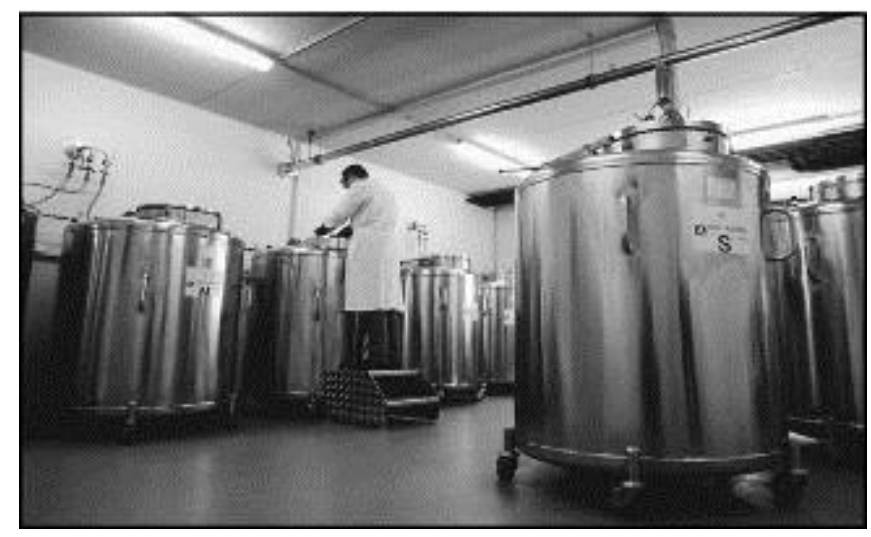

Figure 10 Les caves de stockage.

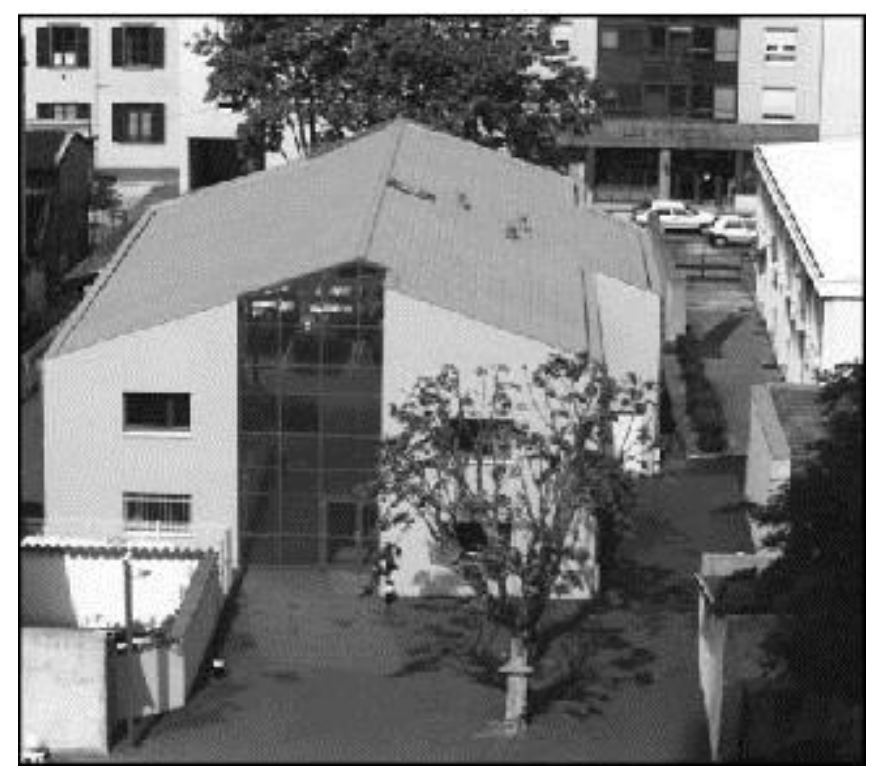

Figure 11 Bâtiment de stockage des cuves (IARC, Lyon).

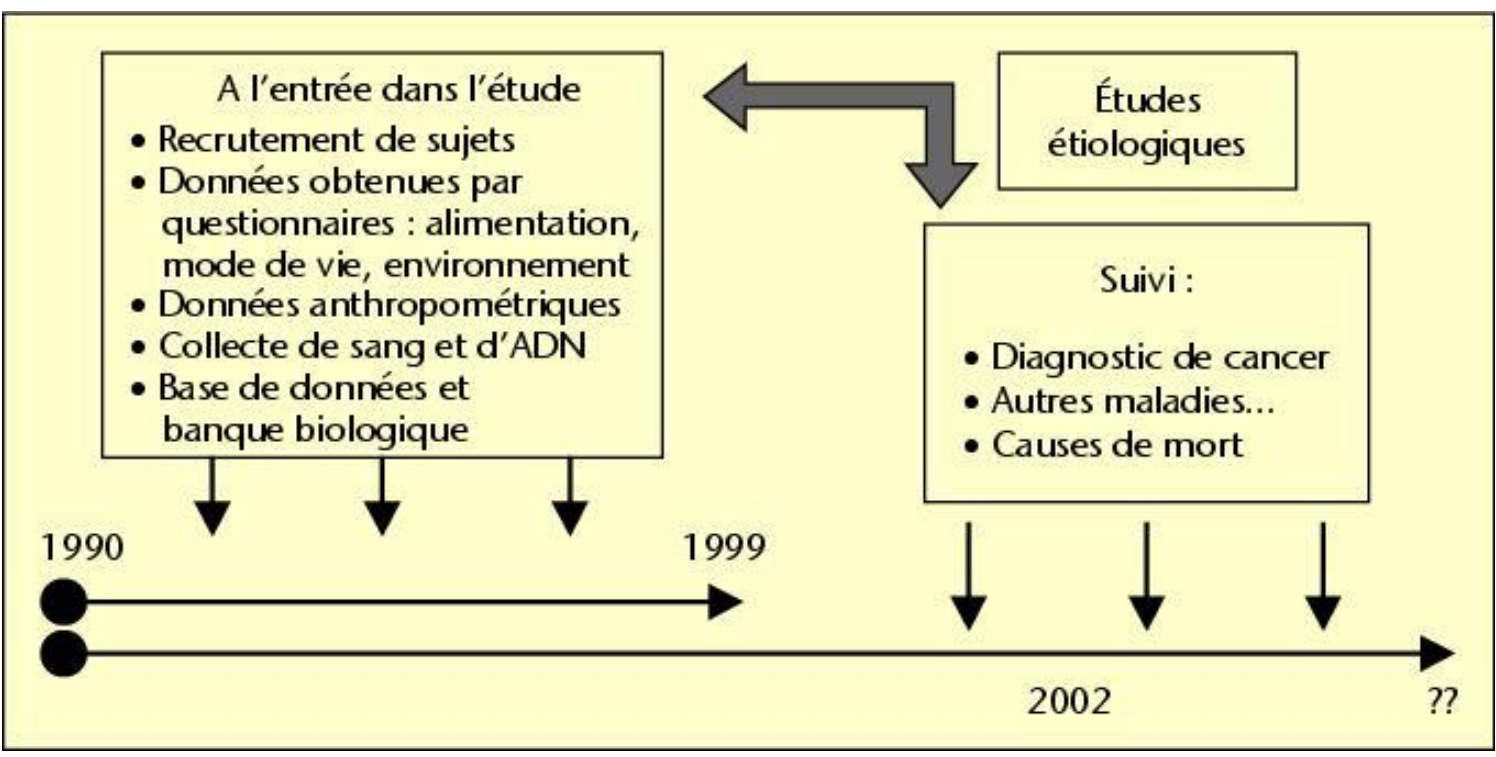

Figure 12 L'étude prospective. 


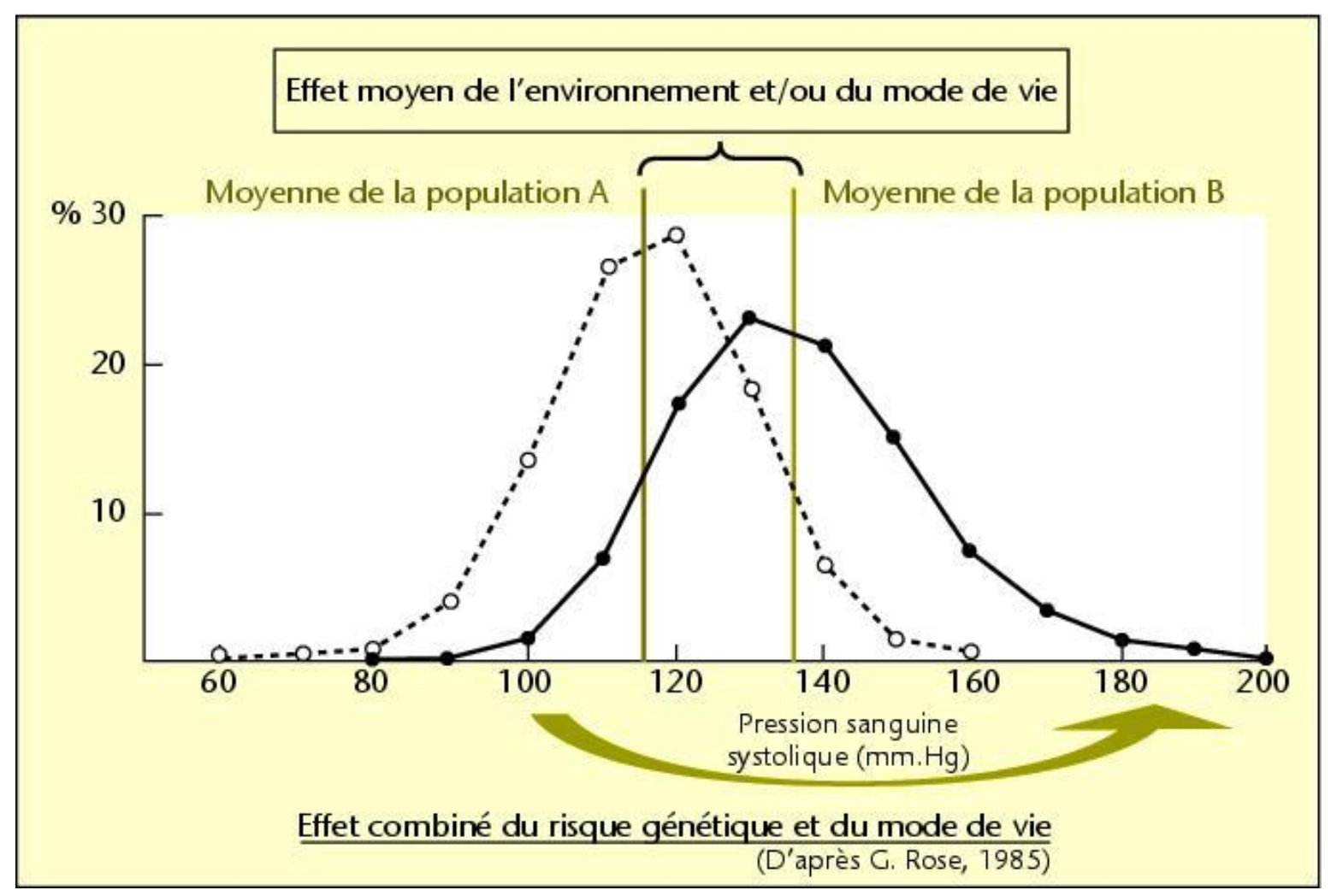

Figure 13 L'approche alternative de G. Rose.

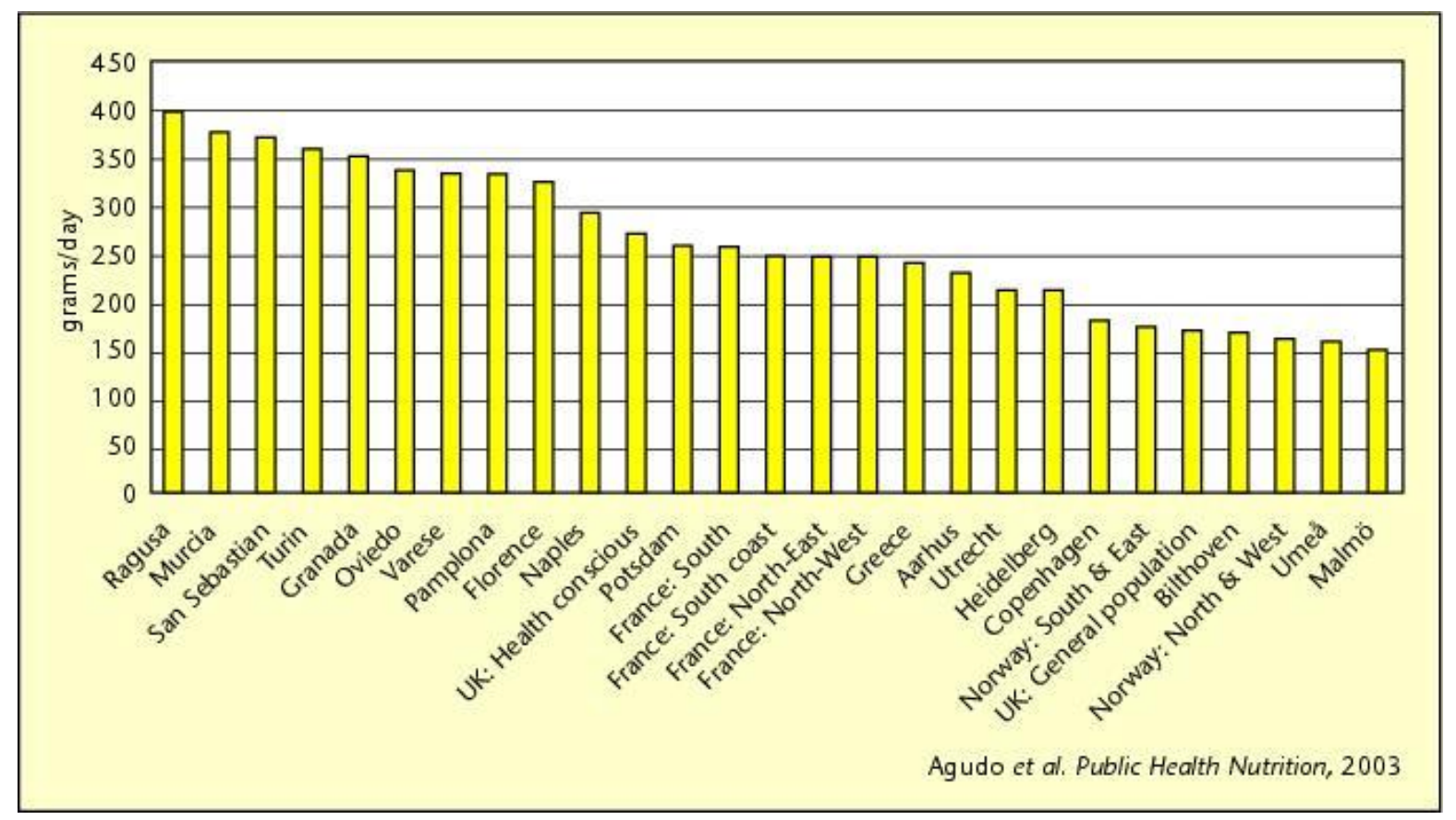

Figure 14 Etude EPIC : consommation journalière de fruits (g/j), sujets féminins. 


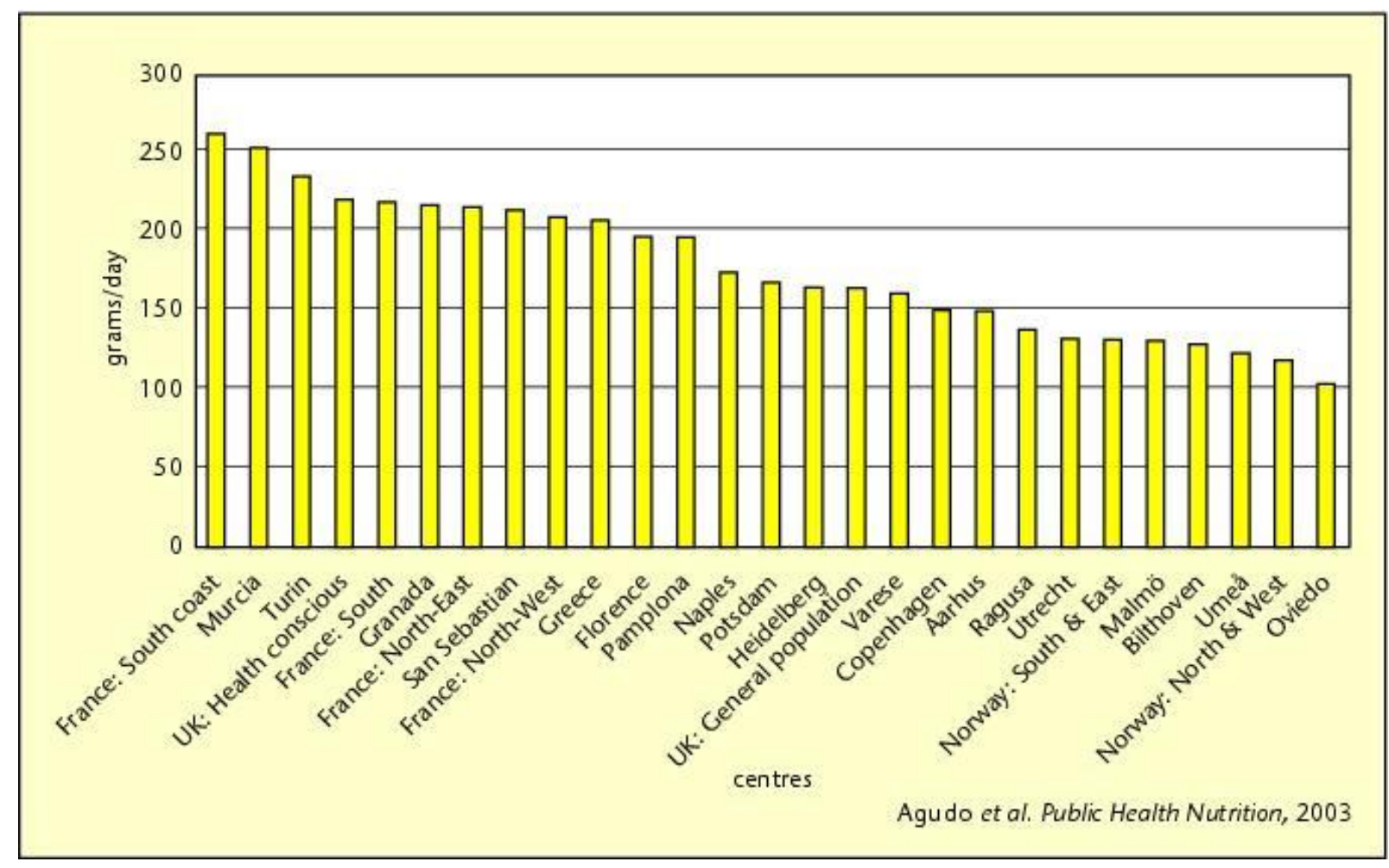

Figure 15 Etude EPIC : consommation journalière de légumes $(\mathrm{g} / \mathrm{j})$, sujets féminins.

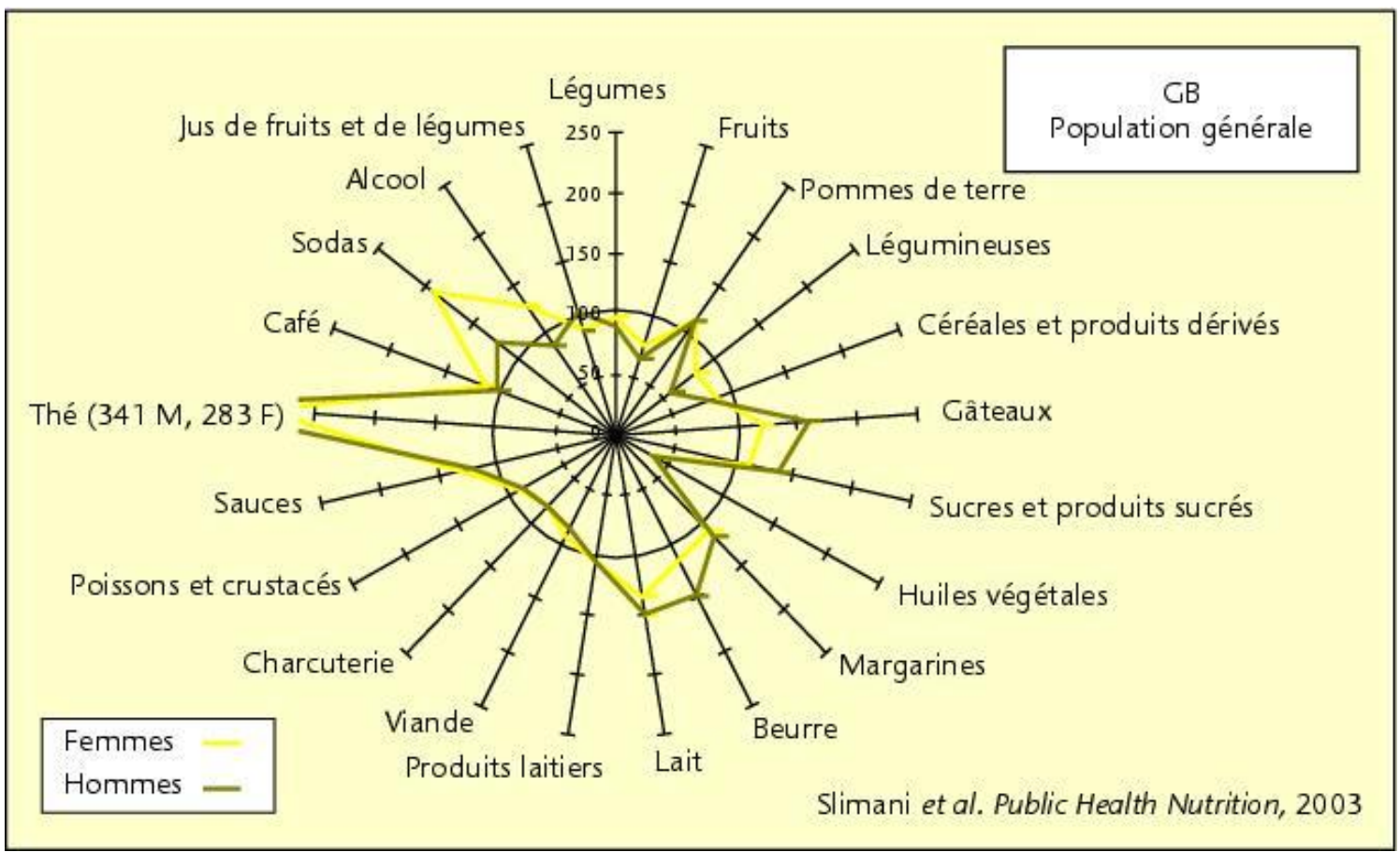

Figure 16 Etude EPIC : comportements alimentaires des sujets britanniques inclus dans la cohorte. 


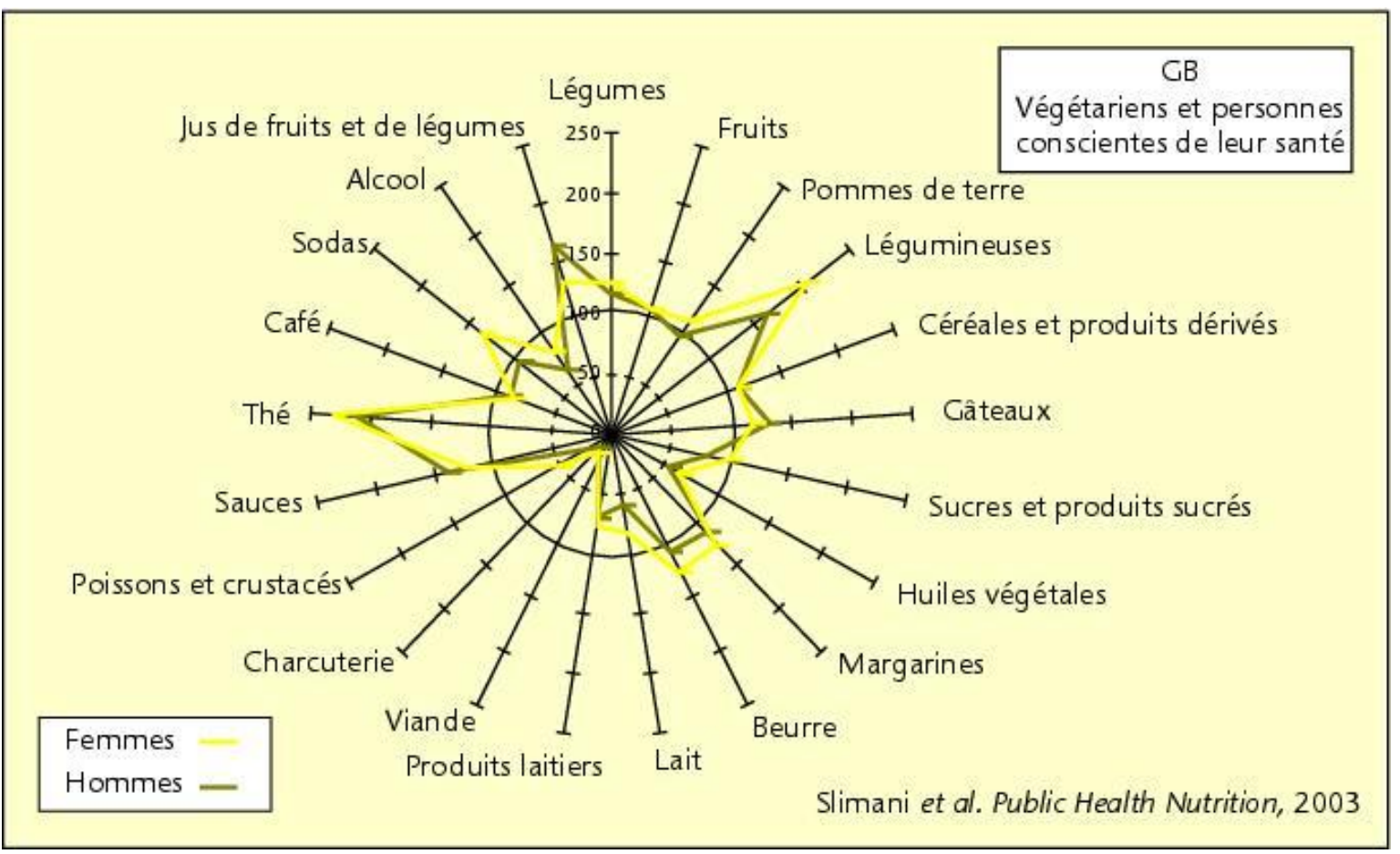

Figure 17 Etude EPIC : comportements alimentaires des sujets britanniques végétariens inclus dans la cohorte.

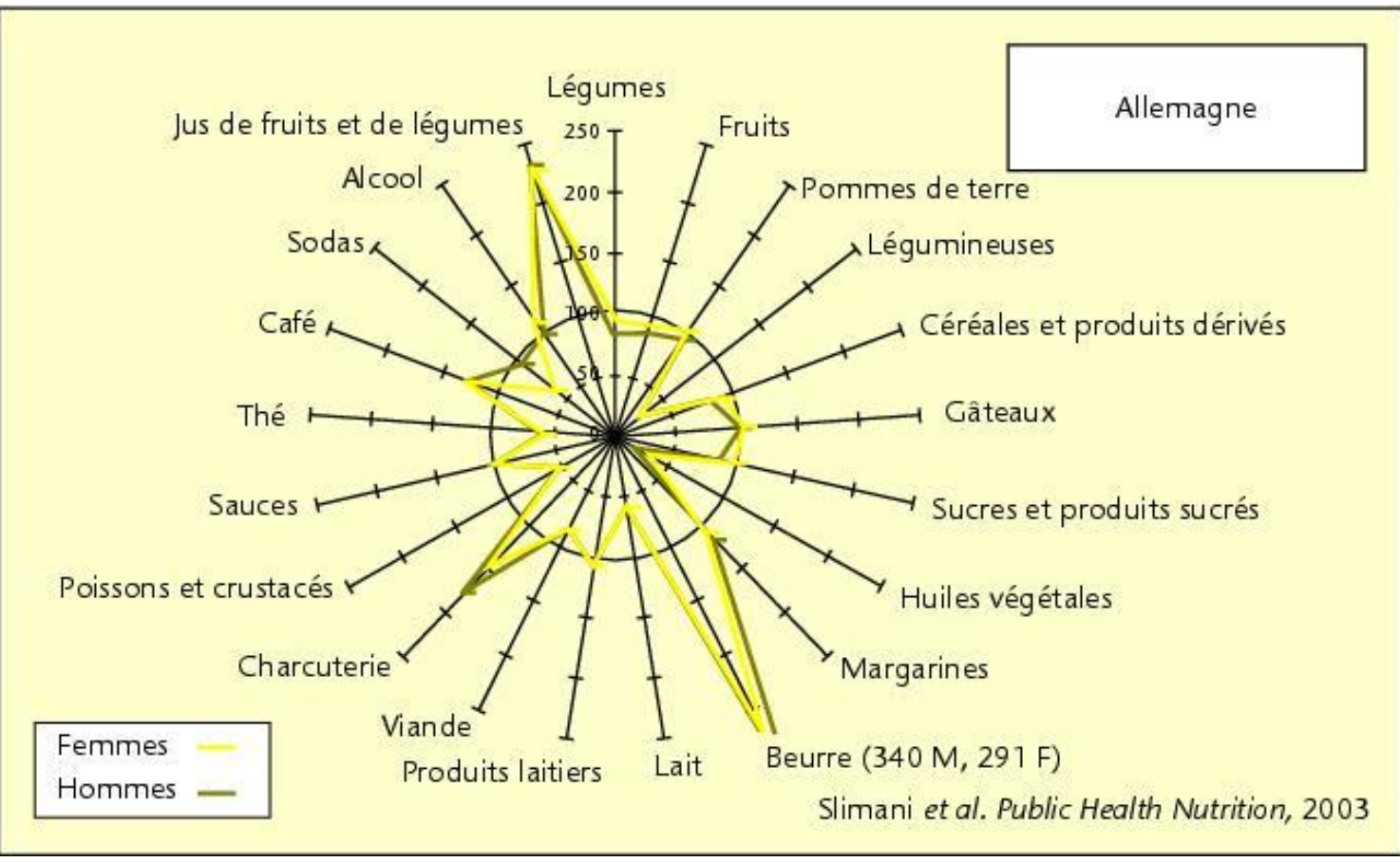

Figure 18 Etude EPIC : comportements alimentaires des sujets allemands inclus dans la cohorte. 


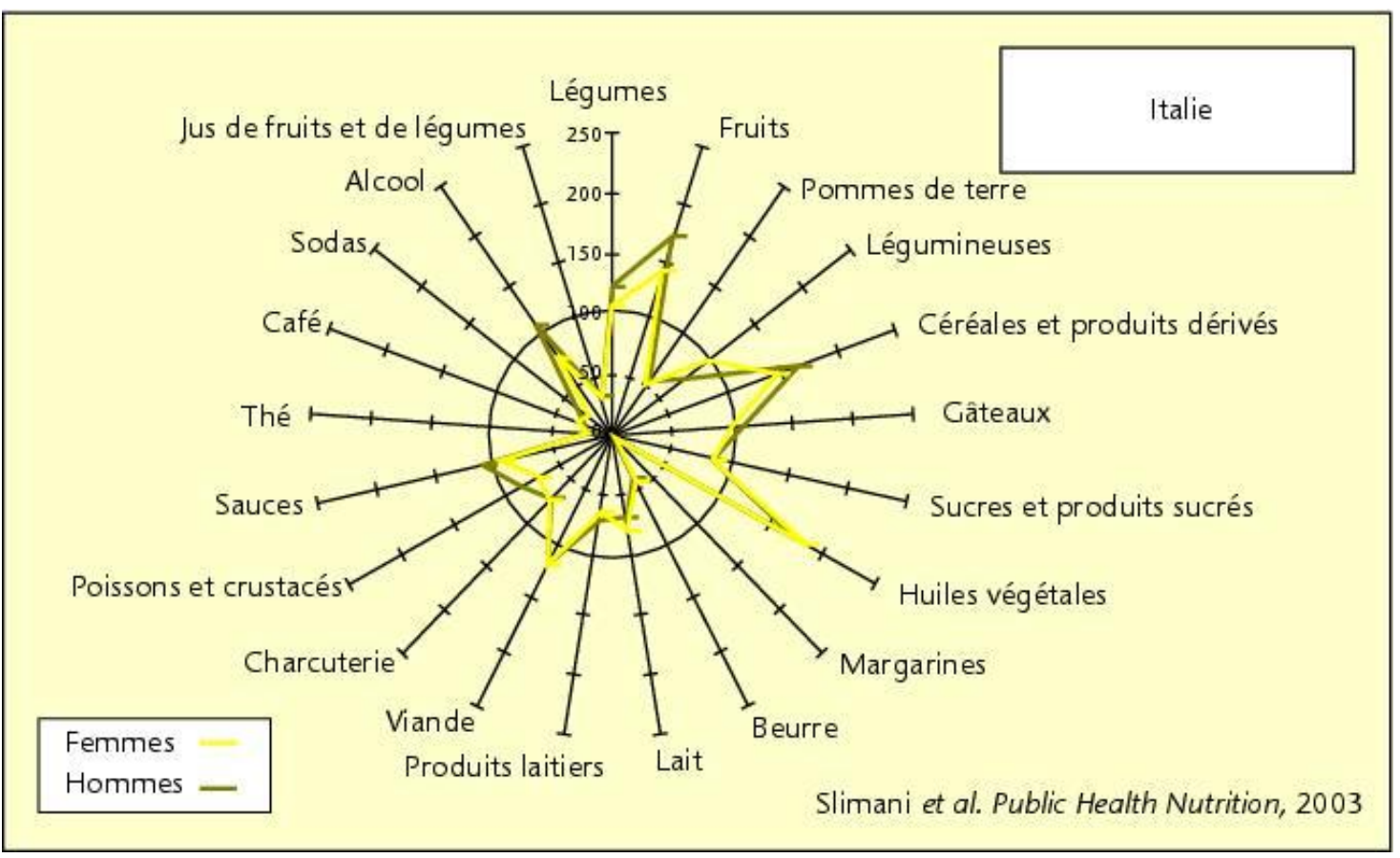

Figure 19 Etude EPIC : comportements alimentaires des sujets italiens inclus dans la cohorte.

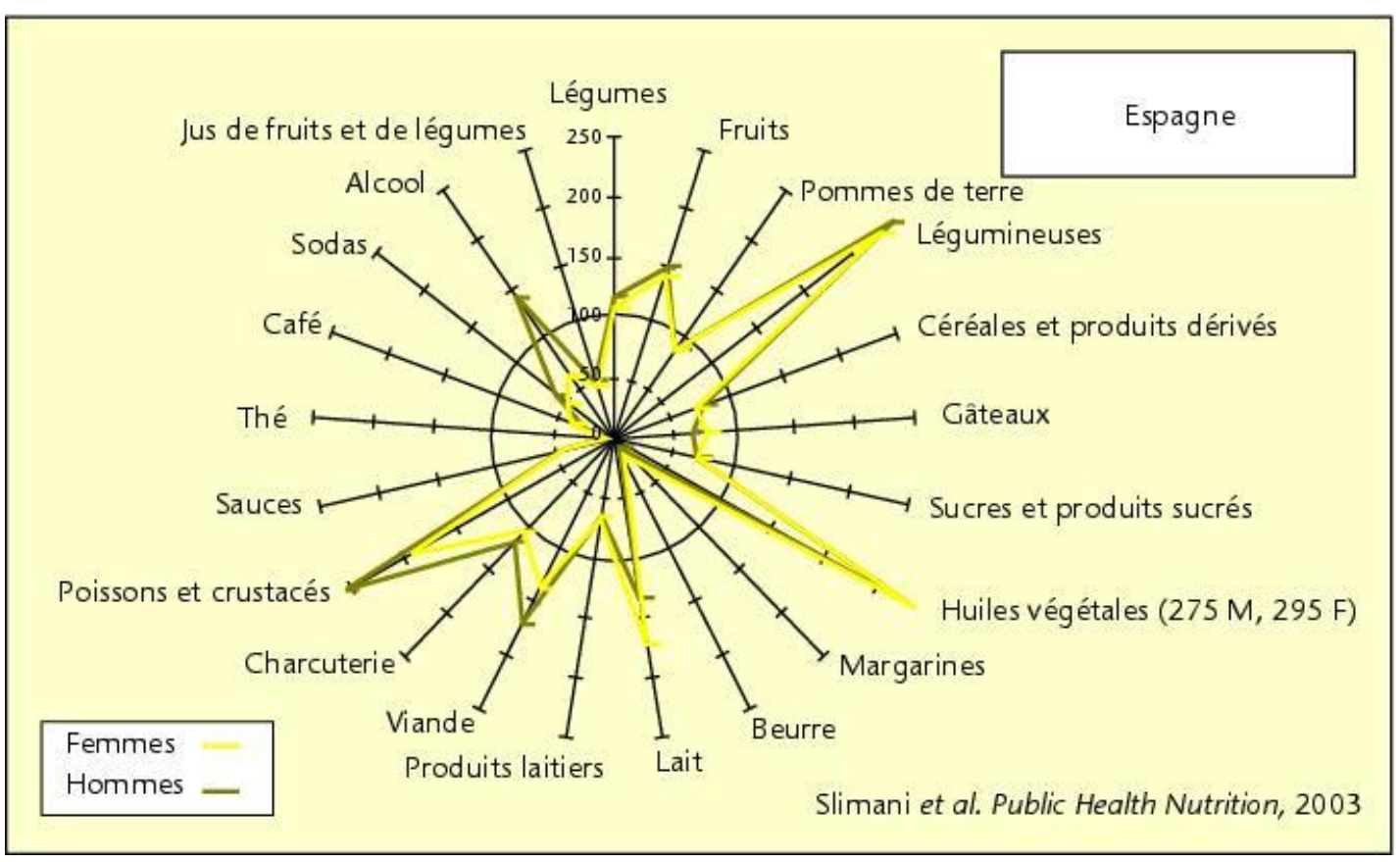

Figure 20 Etude EPIC : comportements alimentaires des sujets espagnols inclus dans la cohorte. 


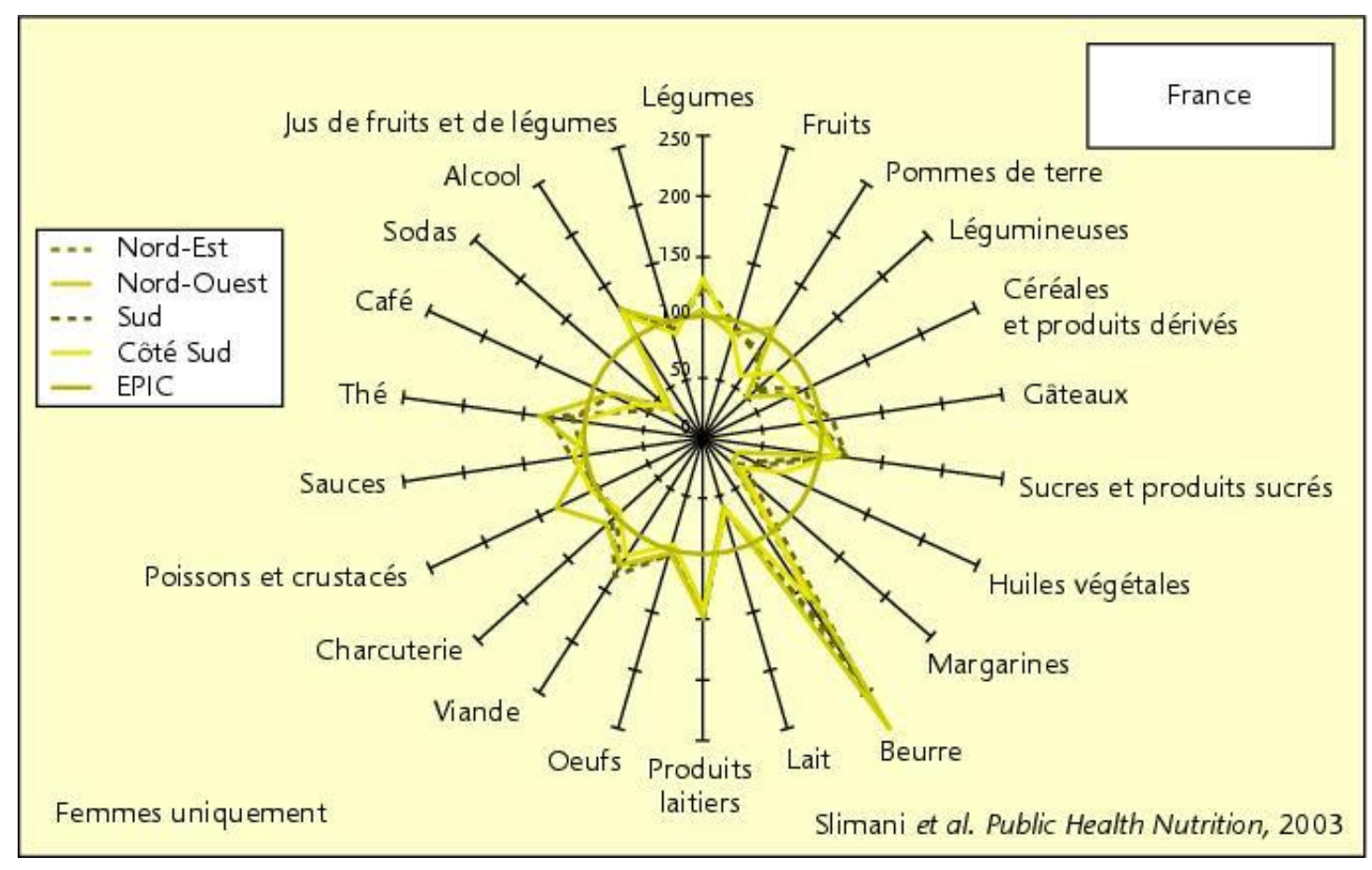

Figure 21 Etude EPIC : comportements alimentaires des sujets français inclus dans la cohorte.

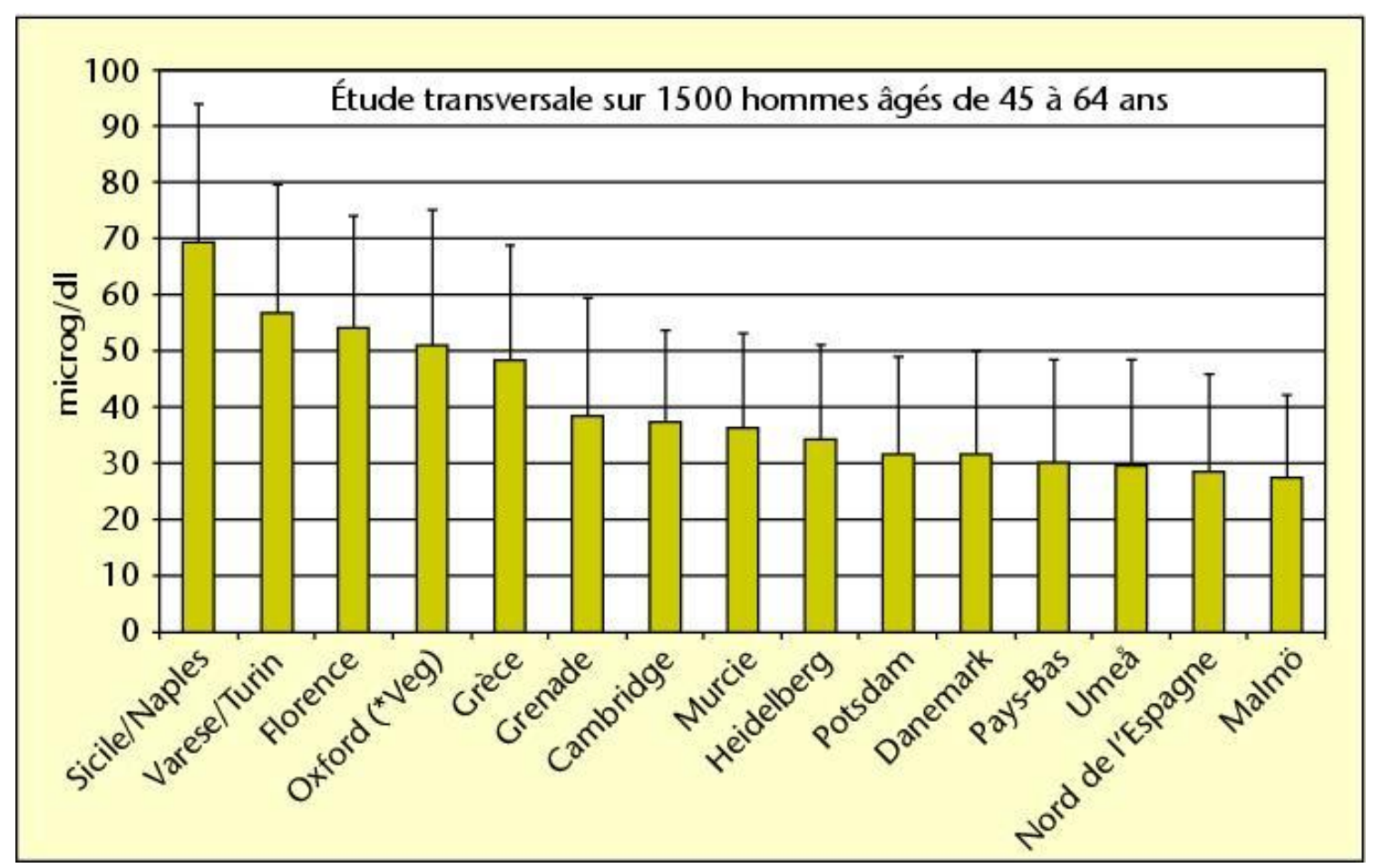

Figure 22 Etude EPIC : variation des biomarqueurs dans la cohorte : cas du lycopène. 


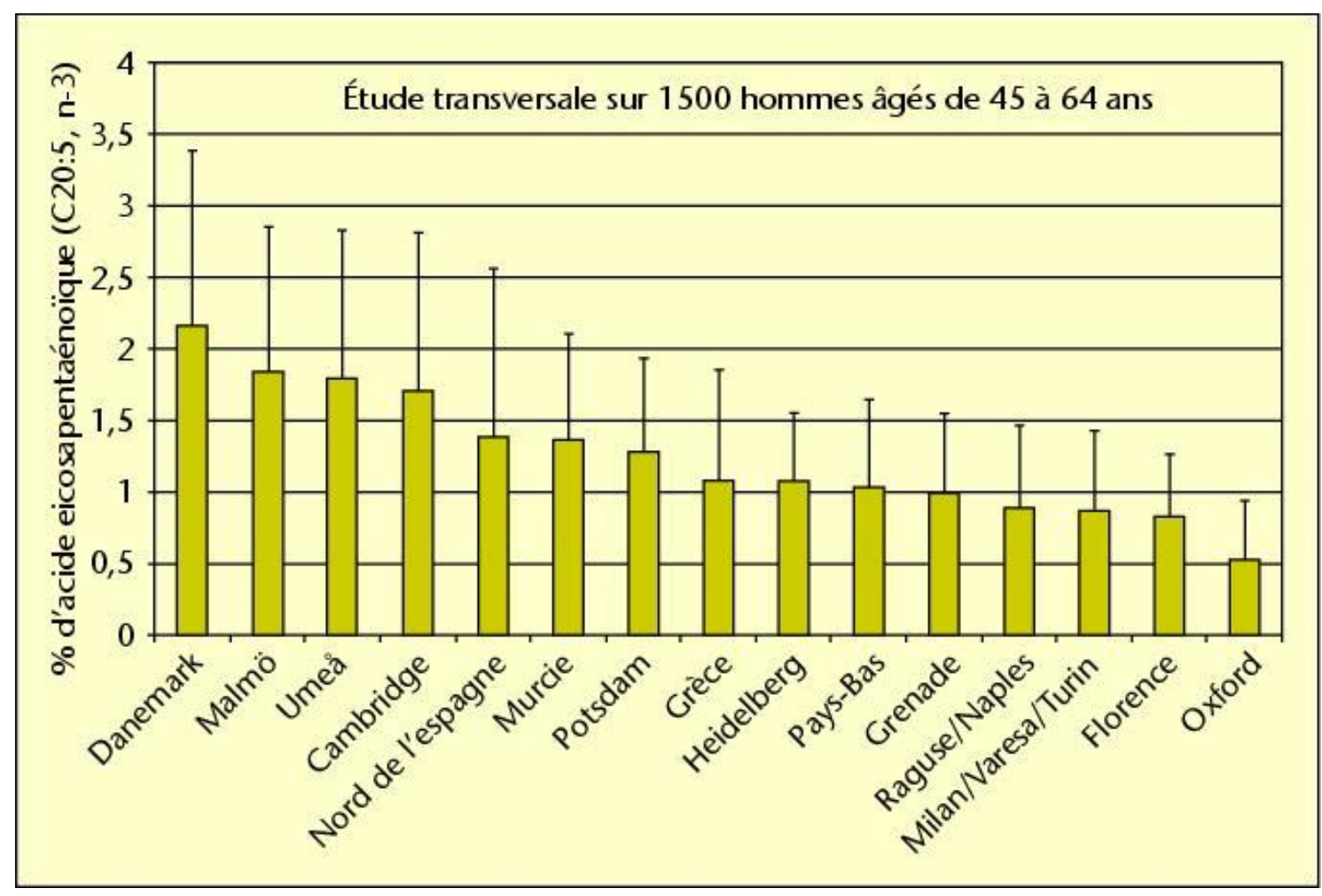

Figure 23 Etude EPIC : variation des biomarqueurs dans la cohorte : cas de l'acide eicospentaénoïque (EPA).

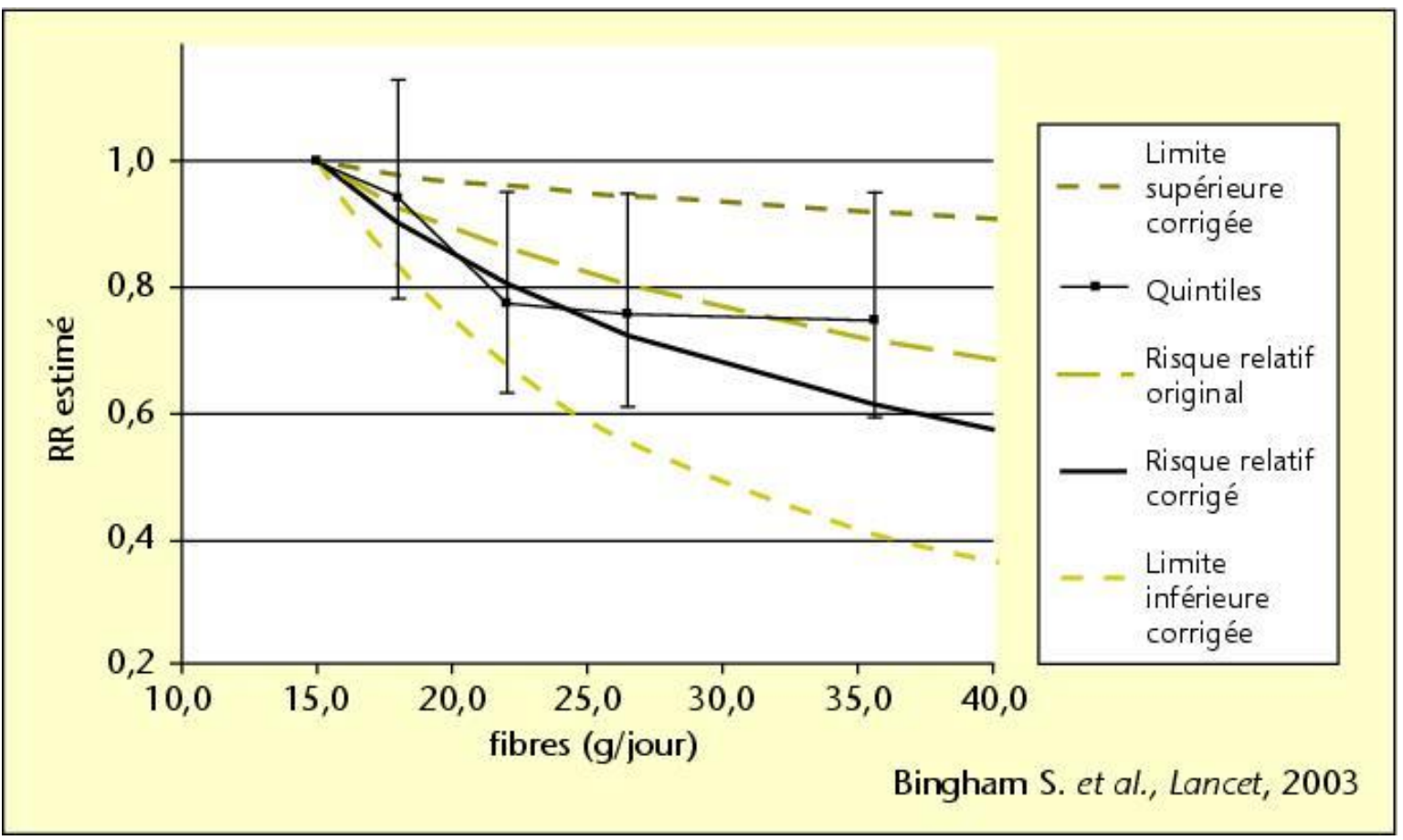

Figure 24 Etude EPIC : consommation de fibres et risque du cancer colorectal. 


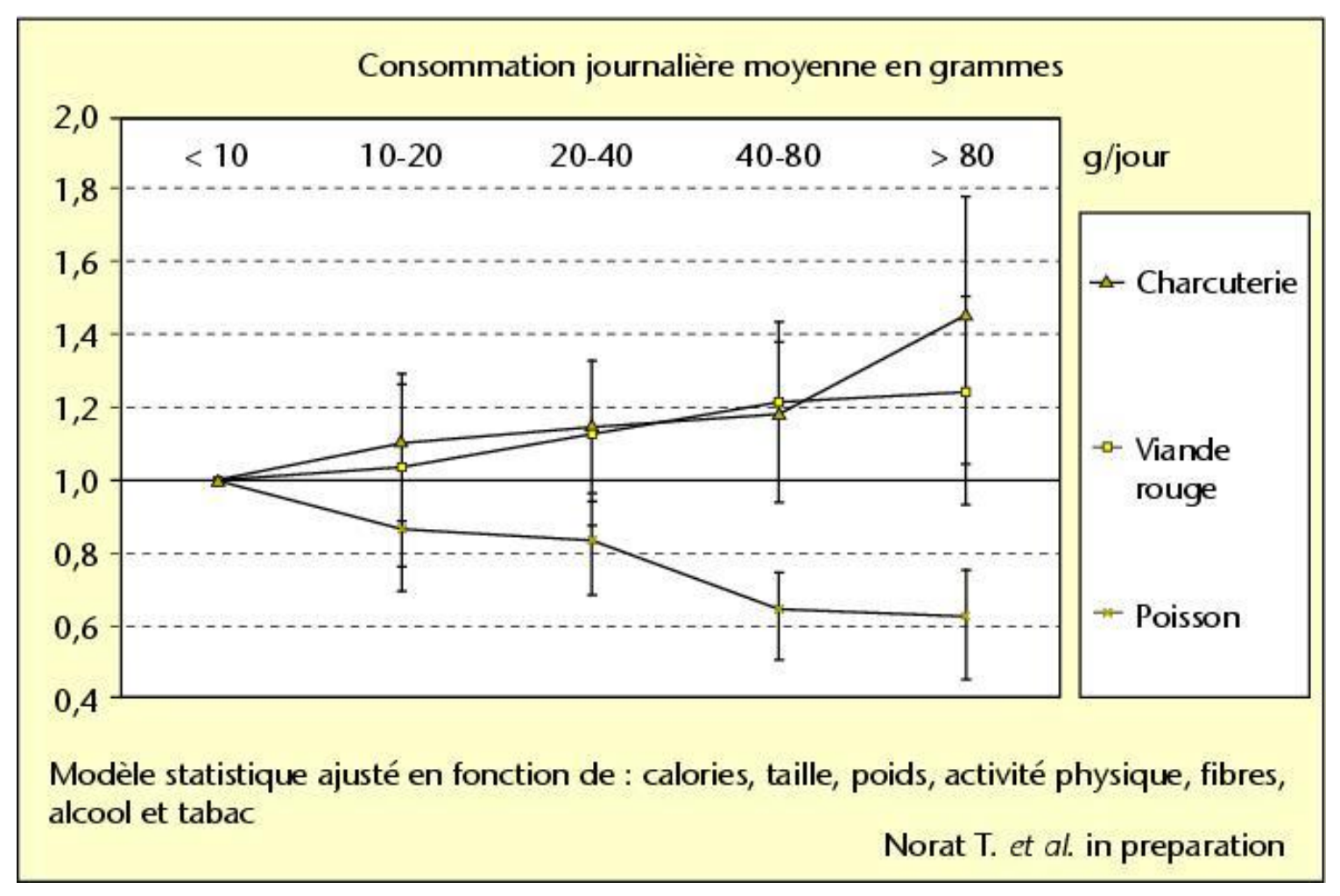

Figure 25 Etude EPIC : consommation de poisson, de viande rouge et risque du cancer colorectal. 